\title{
Morphological and DNA barcode species identifications of leafhoppers, planthoppers and treehoppers (Hemiptera: Auchenorrhyncha) at Barrow Island
}

\author{
David Gopurenko1,3, Murray Fletcher ${ }^{2,3}$, Holger Löcker ${ }^{2}$ and Andrew Mitchell ${ }^{4}$
}

\author{
${ }^{1}$ NSW Department of Primary Industries, Wagga Wagga Agricultural Institute, Pine Gully Rd, \\ Wagga Wagga, New South Wales 2650, Australia \\ 2 NSW Department of Primary Industries, Orange Agricultural Institute, Orange, 1447 Forest Rd, \\ New South Wales 2800, Australia \\ ${ }^{3}$ Graham Centre for Agricultural Innovation (an alliance between Charles Sturt University and NSW \\ Department of Primary Industries), Wagga Wagga, New South Wales 2678, Australia
}

${ }^{4}$ Australian Museum, 6 College Street, Sydney, New South Wales 2010, Australia

\begin{abstract}
The hemipteran suborder Auchenorrhyncha comprises a rich assemblage of plant feeding species, many of which are widespread in distribution and act as vectors of viral and fungal diseases affecting plants. Species level identifications in this group generally are possible only by examination of male specimens; prior DNA barcode analyses of a limited range of Auchenorrhyncha indicate that this approach may provide an expedient means to identify species within this diverse group. In this study we explored the utility of DNA barcoding for identification of a wider range of Auchenorrhyncha species than has been examined previously. Diverse fulgoroid (planthopper) and membracoid (leafhopper and allies) Auchenorrhyncha were sampled from Barrow Island, Western Australia, and identified to the least inclusive taxonomic units using morphology. DNA barcodes from 546 adult specimens were obtained and analysed using a General mixed Yule - Coalescent (GMYC) modelling approach to genetically delimit putative species, as a comparison to the morphospecies identifications. Additional DNA barcodes ( $N=$ 106) were obtained from nymphs and these were compared to adult DNA barcodes to identify species present among immature specimens.
\end{abstract}

Among adult specimens, 73 species were congruently delimited by morphology and genetic analyses when modelled using a single threshold GMYC. Congruence between morphological and molecular species assignments was greatly reduced when the Yule - Coalescent transition was allowed to vary across genetic lineages. In a separate DNA barcode analysis of all specimens using neighbour joining distance metrics, nymphs and physically degraded specimens were in most cases genetically linked to adult conspecifics. Ten genetic clades detected among the nymphs were not observed among adults and did not match pre-existing sequence accessions in GenBank or DNA barcode records in BOLD.

Of the 73 adult Auchenorrhyncha species congruently identified by DNA barcoding and morphology, most were Cicadellidae ( $N=53$ morphospecies), the remaining 20 morphospecies were sparsely representative of ten other families. Formal identifications to species level were available for only $36 \%$ of these 73 morphospecies, owing mainly to an absence of diagnostic male specimens within many of the delimited species. Indeterminate species detected among adults and nymphs are designated with interim species codes.

The work presented here demonstrates that DNA barcoding is likely to be a powerful investigative tool for identifying and understanding species limits in the Auchenorrhyncha, particularly if it is used within an integrative taxonomic framework.

KEYWORDS: mitochondrial DNA, Cytochrome Oxidase I, Cicadellidae 


\section{INTRODUCTION}

The Auchenorrhyncha are a species rich suborder within the megadiverse order Hemiptera comprising cicadas (Cicadoidea), leafhoppers and treehoppers (Membracoidea), planthoppers (Fulgoroidea), froghoppers and spittle bugs (Cercopoidea). All Auchenorrhyncha are plant feeders and many are vectors of viral and fungal diseases of plants. Species level identifications using traditional taxonomic approaches are mainly reliant on examination of the male genitalia. Identifications of females and nymphs are generally restricted to the genus level unless specimens are closely associated with adult males. As a consequence, traditional species delimitation and identification of Auchenorrhyncha is likely to be restrictive under circumstances where rapid bioinventories are required. Given the prevalence of pathogen vector association among many of the Auchenorrhyncha, and the importance of this to global agricultural plant-biosecurity, it is essential that the provision of species-level diagnostics for this suborder is accelerated.

The promise of DNA barcoding as a standardised method to provide rapid and accurate species level identification has been widely touted since its first report early this century (Hebert et al. 2003). DNA barcoding is now a global scientific enterprise aiding taxonomy and species discovery. The premise of DNA barcoding is simple: the majority of species may be identified genetically using their unique nucleotide sequence for a standardised genomic region(s). In the case of animals, the standard diagnostic target is a $>500$ base pair portion of the 5'-portion of the mitochondrial Cytochrome Oxidase I (COI) gene (Hebert et al. 2003). One major criticism of DNA barcoding, as a method for species identification and discovery, concerns the lack of universal operational criteria for assigning a specimen barcode to a given species. Original genetic distance methods which employ empirically determined distance criteria for species delimitation (Hebert et al. 2003), have been largely successful in most cases but are criticised as computationally naïve regarding macro-evolutionary processes (Will and Rubinoff 2004) and vulnerable to error depending on metrics used (Meier et al. 2008) and the extent of congeneric sampling employed (Jansen et al. 2009). Alternative approaches of species delimitation using DNA barcode data include character based analyses to detect parsimonious sharing of species diagnostic nucleotides (Davis and Nixon 1992; DeSalle et al. 2005) and more recent theory-based statistical approaches which employ population coalescent modelling to predict species boundaries from single or multi-locus data (Pons et al. 2006; Rosenberg 2007; Cummings et al. 2008; Yang and Rannala 2010). These new approaches are likely to increase analytical rigour in future DNA barcoding surveys (Fujita et al. 2012).

DNA barcoding has been used as an investigative tool for species delimitation and identification for several families of Hemiptera (Park et al. 2011a, 2011b); however its application to the Auchenorrhyncha largely has been restricted to analyses of cicadellid leafhoppers sampled from Japan (Kamitani 2011) and several significant genera present in the Holarctic (Bluemel et al. 2011; Seabra et al. 2010). Multi-gene analyses of species relationships incorporating the DNA barcode region have also been reported for some significant Auchenorrhyncha present in the Palaearctic (Maryańska-Nadachowska et al. 2010) and in Polynesia (Bennet and O'Grady 2012).

In this study we used DNA barcoding to assist in species identifications of two Auchenorrhyncha superfamilies (Fulgoroidea and Membracoidea) sampled from Barrow Island, Western Australia. Adults were first sorted from immature specimens and identified to morphospecies. We then provided DNA barcode based species delimitations at the adult specimens and compared these to their morphospecies determinations to assess the extent of congruence between the two independent approaches to species inventory. We used a recently developed method of genetic species delimitation to analyse the DNA barcodes, which identifies terminal genetic species based on modelling of expected differences in phylogenetic branching between population coalescence and species diversification (Pons et al. 2006; Monaghan et al. 2009). In addition, we used DNA barcoding to provide putative species identities for morphologically indistinct nymph specimens. For this, we used simple pair-wise genetic distance methods to distinguish immature specimens and to provide putative species identities to them based solely on their DNA barcode match to adults.

\section{METHODS}

\section{SAMPLING AND LABORATORY ANALYSES}

Adult $(\mathrm{N}=672)$ and nymph $(\mathrm{N}=106)$ Auchenorrhyncha were sampled at 26 sites during 2005-2007 as part of a baseline survey of terrestrial invertebrates for the Gorgon Gas Development on Barrow Island, Western Australia (Callan et al. 2011). Samples were transferred to the NSW Agricultural Scientific Collections Unit (Orange Agricultural Institute, NSW Department of Primary 
Industries) for taxonomic identifications and storage. Adult specimens were morphologically sorted and identified to the least inclusive taxonomic level based on male genitalia.

In preparation for DNA extraction, adult specimens' abdomens or legs, and whole specimen nymphs were non-destructively digested overnight at $55^{\circ} \mathrm{C}$ in separate aliquots containing $360 \mu \mathrm{l}$ of tissue digestion buffer (QIAGEN, Doncaster, Australia) and $40 \mu \mathrm{l}$ of proteinase-K solution (QIAGEN) diluted to a final volume of $1 \%$. DNA extractions were conducted using a Corbett Research 1820 X-tractor Gene robot with recommended protocols and DNA extraction kit reagents (QIAGEN). Final DNA elutions of $120 \mu \mathrm{l}$ were stored at $-20^{\circ} \mathrm{C}$. Mitochondrial cytochrome oxidase I (COI) DNA barcodes were targeted for amplification by polymerase chain reaction (PCR) using primer $\mathrm{BC} 1 \mathrm{Fm}$ in combination with either BC3RDm or JerR $2 m$ (Table 1) yielding PCR amplicons of lengths 672 $\mathrm{bp}$ and $646 \mathrm{bp}$, respectively (all reported amplicon lengths exclude primer sequences). Overlapping short fragment PCRs were attempted when fulllength DNA barcodes failed to amplify (Table 1). For this, the 5'-portion of the original COI target amplicon was amplified with primers BC1Fm and Scar-2RDm to give a $328 \mathrm{bp}$ fragment; primers Scar-3aFm and JerR2m were used to amplify the 3'-portion, yielding a $406 \mathrm{bp}$ product. The overlap between these amplicons was $88 \mathrm{bp}$. PCR Primers incorporated a 17 nucleotide M13 vector sequences at their 5 '-ends, to simplify downstream sequencing. PCR amplicons ( $15 \mu \mathrm{l})$ were prepared using a Corbett 1200 PCR robot, and contained 4 $\mu l$ of DNA extract in the presence of Invitrogen reagents: $1 \mathrm{X}$ PCR buffer, $3 \mathrm{mM} \mathrm{MgCl}_{2}, 0.4 \mathrm{U}$ Platinum Taq polymerase, $200 \mu \mathrm{M}$ dNTPs and 2 pmol each of forward and reverse primers. Thermal cycling using an Eppendorf Mastercycler ep gradient
$S$ PCR machine consisted of an initial two minute $94^{\circ} \mathrm{C}$ denature followed by a 40 cycle profile ( 30 seconds denature at $94^{\circ} \mathrm{C}, 30$ seconds anneal at $50^{\circ} \mathrm{C}, 60$ seconds extension at $72^{\circ} \mathrm{C}$ ) ending with a five minutes extension at $72^{\circ} \mathrm{C}$ and storage at $4^{\circ} \mathrm{C}$. PCR products were visualized using a UV trans-illuminator after electrophoresis through a $1.5 \%$ agarose gel in $1 \%$ TAE buffer; products were qualitatively checked for expected fragment size against E-Gel size marker (Invitrogen). PCR products were sent to AGRF (Brisbane) for purification and bidirectional sequencing using M13 sequencing primers.

Forward and reverse AB1 trace files from each DNA extraction were checked for quality using SeqMan Pro ver. 8.1.0(3) (DNASTAR, Inc.) and assembled against a deltocephaline reference sequence (sp. J129, Le Roux and Rubinoff (2009); GenBank accession \# EU981895.1) to generate consensus sequences. Primer sequences were masked at the assembly stage. Alignment of consensus sequences in preparation for genetic analyses was conducted using BioEdit ver. 7.0.5.3 (Hall 1999). All sequences and trace files, as well as specimen images and sample data, were uploaded to the Barcode of Life Data System (BOLD) (Ratnasingham and Hebert 2007) and are publically available under the project "Barrow Island Hemiptera" (project code: BIH), sequences are also available at GenBank (accessions KF226727KF227378).

\section{DNA BARCODE ANALYSIS AND SPECIES DELIMITATIONS OF ADULTS}

Sequence analysis of adult DNA barcodes followed a 2 step procedure.

Step 1 identified putative species from DNA barcodes using a statistical approach (Pons et al. 2006; Monaghan et al. 2009) to detect shifts in

TABLE 1 Primers used for amplification of partial mitochondrial Cytochrome Oxidase I gene product. Refer to Methods for primer combinations used and PCR conditions. Seventeen base pair M13-vector sequence 5 '-tails are italicised and underlined in forward and reverse primer directions respectively. Primer sources: (1) Cho et al. (2008) and modified from (a) Folmer et al. (1994); (2) modified from forward primer ("Jerry" of Simon et al.1994); (3) new primer.

\begin{tabular}{llc} 
Primer & Sequence $\left(\mathbf{5}^{\prime}-\mathbf{3}^{\prime}\right)$ & Source \\
\hline BC1Fm & GTAAAACGACGGCCAGTCwACwAAyCAyAArGAyATyGG & $1 \mathrm{a}$ \\
Scar-3aFm & GTAAAACGACGGCCAGGChCChGAyATAGCnTTyCCnCG & 3 \\
BC3RDm & CAGGAAACAGCTATGACCChGArGTwTAyATTyTwATTyTwC & 1 \\
JerR2m & CAGGAAACAGCTATGACCArCAyyTrTTyTGrTTyTTTGG & 2,3 \\
Scar-2RDm & CAGGAAACAGCTATGACGArArwGGnGGrTAnACwGTTC & 3 \\
\hline
\end{tabular}


lineage branching rates distinguishing species divergence (Yule 1924) from population coalescent processes (Kingman 1982). Application of a general mixed Yule - coalescent (GMYC) model fitted to a clock-constrained gene tree can be used to identify the threshold at which there is a substantial rate shift in lineage accumulation demarcating species divergence from intraspecific diversification (Pons et al. 2006). Nodes occurring before the threshold identify earlier species diversification events; nodes after the threshold identify intraspecific population coalescence events (Pons et al. 2006; Papadopoulou et al. 2008; Monaghan et al. 2009). In preparation for this analysis, adult sequences less than 500 bp were discarded and the remaining sequences were truncated to an equal length alignment using BioEdit ver. 7.0.9.0 (Hall 1999). Identical sequences in the edited alignment were identified and collapsed as unique haplotypes using FaBox ver.1.35 (Villesen 2007). A maximum likelihood ultrametric tree incorporating a uniform molecular clock, and a General Time Reversible nucleotide substitution model with Gamma distributed site rates $(G T R+G)$, was generated from the haplotype alignment using PAUP* ver. 4.0b10 (Swofford 1998) and seeded by an initial UPGMA tree. The GTR+G model and parameters used in the ML tree search were initially determined as a best fit of the data using jModelTest (Posada 2008). A single threshold general mixed Yule coalescent (GMYC) model was optimised at the ultrametric tree to identify location of the threshold separating speciation from coalescent events, using GMYC script implemented in the Splits package for R (Pons et al. 2006; http://rforge.r-project.org/projects/splits/) and functions within the APE library for R (Paradis et al. 2004). The predicted speciation - coalescent threshold was mapped onto a lineage through time (LTT) plot that was constructed as a cumulative frequency curve of inter-nodal branch occurrence observed within the ultrametric tree. A likelihood ratio test comparing the GMYC optimisation against a null model (where the entire sample is assumed to have a uniform branching rate) was used to test if there was evidence to reject the predicted transition threshold (Monaghan et al. 2009). Lineages observed after the predicted threshold leading to one or more constituent haplotypes were numerically labelled as putative species.

We also explored a multiple threshold GMYC model optimised to the ultrametric tree (Monaghan et al. 2009), and compared genetic species delimitations determined by this latter model with that detected using the single threshold. The multiple threshold GMYC model differs from the single threshold model by allowing recognition of multiple coalescent to speciation transition events within a tree. Effectively it allows for variable rates of lineage evolution among independent clades.

Step 2 compared membership of the genetically delimited putative species identified by the single and mixed GMYC models with those determined by morphological examination. Specimen replicates at haplotypes within each genetic species were retrospectively examined to determine if they shared the same morphospecies identification. Summary information on genetic differences within and between species congruently defined by morphology and genetics was calculated from species haplotypes using MEGA4 (Tamura et al. 2007). For these calculations, only haplotypes $>500$ $\mathrm{bp}$ of comparable sequence were used.

\section{NYMPH SAMPLE SEQUENCE ANALYSIS}

Nymph sequences $(\mathrm{N}=106)$ were compared to all adult sequences (including sequences $<500 \mathrm{bp}$ ) to determine whether nymphs could be assigned to species using DNA barcodes. A neighbour joining tree (Saitou and Nei 1987) was calculated using the Kimura 2-parameter distance model (Kimura 1980) with node support estimated by bootstrapping $(1,000$ replicates) as implemented in MEGA4 (Tamura et al. 2007). Nymphs were identified to species level if they either shared a DNA barcode haplotype with an adult morphospecies or had a novel haplotype which was nested within the clade of an adult morphospecies. Nymphs with novel haplotypes not nested within adult clades were assigned as new putative species not observed among the adult morphospecies and lacking a taxonomic identity. Sequences of these new putative species were queried against GenBank (on 20 Oct. 2012) using the standard nucleotide BLAST algorithm at NCBI (http://www.ncbi.nlm.nih.gov/). Accession records matching sequences with $>97 \%$ sequence similarity (at $>95 \%$ sequence coverage) were considered a positive match at the species level. Similar query searches were conducted using the species identification tool at BOLD.

\section{RESULTS}

\section{SPECIES DELIMITATION}

Adult specimens were morphologically delimited into 73 taxa (Table 2). These included 26 formally described species, 45 indeterminate species and two taxa that could not be adequately identified below the level of family due to sample condition. Many of the indeterminate taxa could not be positively identified to species level as 
(A)

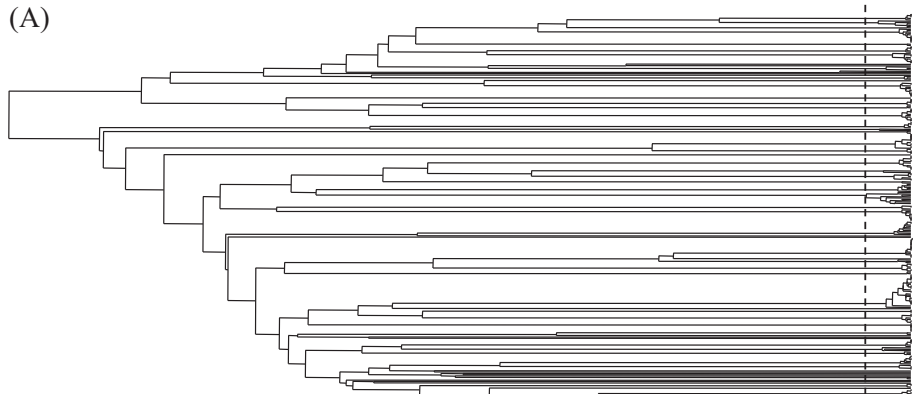

(B)

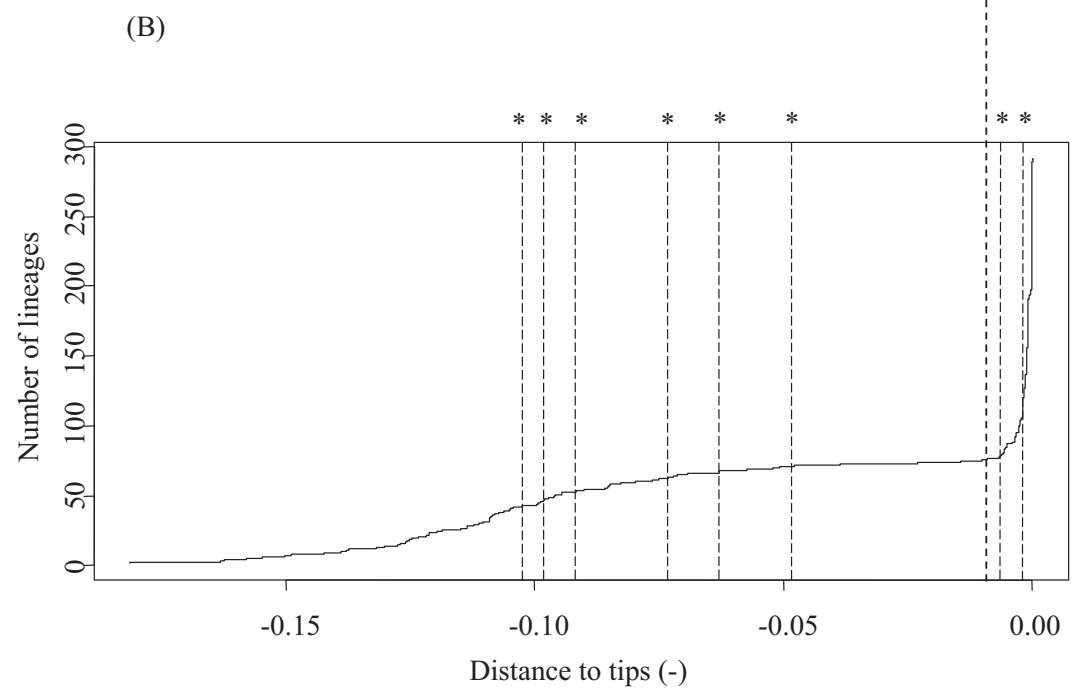

FIGURE $1 \quad$ Ultrametric clock constrained maximum likelihood tree of 291 unique haplotypes identified among Barrow Island Auchenorrhyncha (A); and corresponding lineage through time (LTT) plot (B). The vertical dashed line spanning $(A)$ and $(B)$ indicates the speciation - coalescent transition in branching rate identified using a single threshold general mixed Yule-Coalescent (GMYC) model. Eight additional transition events identified using a multiple threshold GMYC model indicated in the LTT plot by dashed lines and *. Distance to tips is scaled from -1 (base of the tree) to 0 (terminal haplotypes).

they were represented by female specimens only. The remaining indeterminate males could not be identified to taxa currently recorded from Australia.

PCR products were successfully recovered from $546(81.3 \%)$ of the 672 adult samples. Of these, DNA barcode sequences $>500$ bp were obtained from 478 samples; the remaining 68 samples were recovered as partial DNA barcode sequences ranging in size from 242-499 bp. There was no evidence of nucleotide base insertions/deletions or amino acid stop codons among sequences. The average percentage of $\mathrm{A}$ and $\mathrm{T}$ residues among sequences was $67.3 \%$. A total of 291 unique haplotypes were identified among DNA barcodes $>500 \mathrm{bp}$.
The maximum likelihood ultrametric tree of genetic relationships among the 291 haplotypes was converted as a lineage through time (LTT) plot to show a cumulative frequency curve of inter-nodal branch occurrence (Figure 1). In the LTT plot, two prolonged phases of minimal branch accumulation from the base of the tree were followed by a single steep rate increase of very short duration towards the tips of the tree. The point of increase commencing the final steep phase was indicative of an expected transition between inter and intraspecific rates of lineage branching (Pons et al. 2006). This transition was optimally fitted by the single threshold GMYC model as occurring at -0.0092 distance units before present. 
Seventy six putative species were delimited by this single threshold transition (Supplementary Figure 1A), with a $95 \%$ confidence interval (within two log-likelihood units of the maximum likelihood) ranging from 73-76 species. Sixty percent of the putative species $(\mathrm{N}=46)$ were represented by multiple haplotypes, the remaining species $(\mathrm{N}=30)$ were each represented by a single haplotype. The single threshold GMYC model was a significantly better fit of the data compared to a null model of uniform branching rates $\left(\log \mathrm{L}_{\text {single }}=2640\right.$ vs $\log \mathrm{L}_{\text {null }}$ $=2391 ; 2 \Delta \mathrm{L}=498, \mathrm{P}<0.001$, d.f. $=3$ ). The multiple threshold GMYC model was a significantly better fit of the data compared to both the null model of uniform branching rates $\left(\log \mathrm{L}_{\text {multiple }}=2747.4 \mathrm{vs}\right.$ $\log \mathrm{L}_{\text {null }}=2391 ; 2 \Delta \mathrm{L}=712.9, \mathrm{P}<0.001$, d.f. $=3$ ), and to the single threshold model $\left(\chi^{2}=214.41\right.$, d.f., $=21$, $\mathrm{P}<0.001)$. The multiple threshold GMYC model identified eight Yule - coalescent transitions, none of which overlapped with that seen in the former model (Figure 1B); six transitions were shifted to the base of the tree relative to that seen at the former model, and two were shifted towards the tips. The latter two transitions were intermediate within the most rapid zone of rate change apparent in the LTT plot. In contrast the remaining six transitions were not associated with any prominent visible rate shifts on the plot. Ninety six putative species were delimited by the multiple threshold model (Supplementary Figure 1B), with a 95\% confidence interval ranging from 81-97 species.

Species delimitations using the single threshold GMYC model $(\mathrm{N}=76)$ were highly congruent both in number and sample membership of species delimited with that determined by morphology $(\mathrm{N}=73)$ (Table 2; Appendix 1). In three instances, a morphological species was split as two putative sister species (Sp. Indet. (9) split as genetic species 11 and 12; Orosius argentatus (43) split as genetic species 72 and 73, and O. orientalis (45) split as genetic species 75 and 76). In contrast, the multiple threshold GMYC model identified a greater number of putative species $(\mathrm{N}=96)$ than that identified by either the single threshold model $(\mathrm{N}=76)$ or by morphology $(\mathrm{N}=73)$. Putative species identified using the multiple threshold model showed poor association with species identified either using the single threshold model or by morphological analysis; only 27 species (37\%) were congruently identified by the multiple threshold model and by morphology (Appendix 1). Sixteen morphospecies were split as two or more putative species and thirty morphospecies were fused with others as single putative species.

Identifications of the 73 morphologically defined species and their frequency of occurrence within the sample are seen in Table 2. Eleven Auchenorrhyncha families were represented in the sample (Table 2; Appendix 1). Greatest alphataxonomic diversity was seen in the Cicadellidae, represented by 53 species of leafhoppers (Figure 2); species counts within each of the remaining ten families averaged at two with a maximum of four species of Delphacidae and Nogodinidae. Average sequence distance $( \pm$ S.E.) among haplotypes within morphospecies was $0.31 \%( \pm 0.01)$, with a maximum of $4.66 \%$ for Orosius argentatus. Distances between nearest neighbour species ranged from $7.20-20.87 \%$ with an average of $12.81 \%( \pm 0.12)$. A neighbour joining tree of K2P distances among morphospecies is presented in Figure 3.

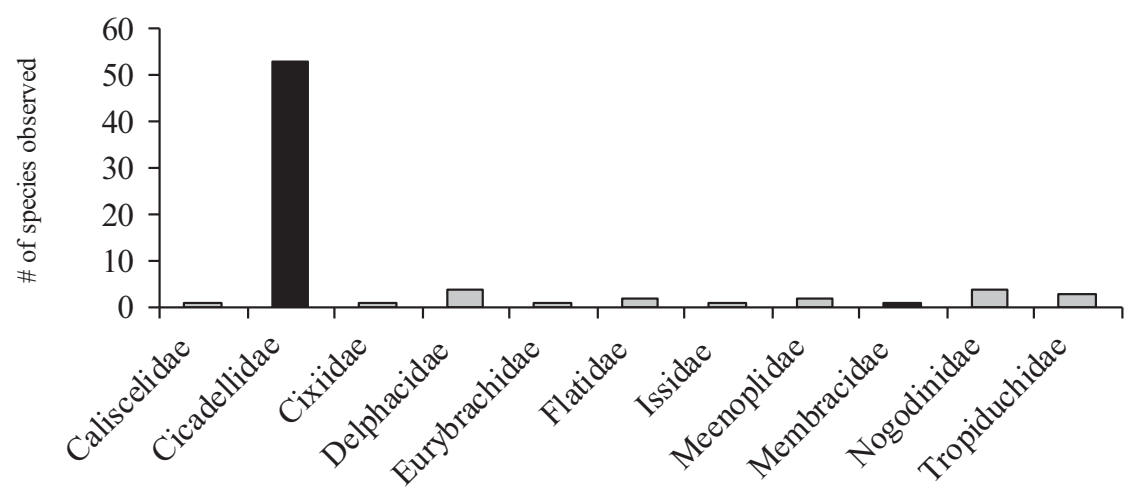

FIGURE 2 Number of morphospecies identified in 10 families of Auchenorrhyncha sampled from Barrow Island (Fulgoroidea shaded in grey; Membracoidea shaded in black). 
TABLE 2 Taxonomy of 73 morphologically identified Barrow Island Auchenorrhyncha, and corresponding 76 genetic species. Adult morphospecies identified to least inclusive taxonomic unit, numbers in parentheses indicate morphospecies numerical designation (1-73). Genetic species (1-76) delimited using a single threshold GMYC model analysis applied to an ultrametric maximum likelihood tree of sequenced DNA barcode haplotypes. Specimens sorted numerically by genetic species designation. $N=$ number of samples identified to each morphospecies. Adult specimen affiliation at each morpho and genetic species detailed in Appendix 1.

\begin{tabular}{|c|c|c|c|c|c|}
\hline Family & Subfamily & Tribe & Morphospecies & $\begin{array}{l}\text { Genetic } \\
\text { species }\end{array}$ & $\mathrm{N}$ \\
\hline Nogodinidae & Nogodininae & Lipocalliini & Lipocallia sp. (17) & 1 & 7 \\
\hline Nogodinidae & Nogodininae & Lipocalliini & Lipocallia sp. (16) & 2 & 7 \\
\hline Nogodinidae & Nogodininae & Lipocalliini & Bilbilicallia sp. (18) & 3 & 3 \\
\hline Nogodinidae & Nogodininae & Lipocalliini & Lipocallia sp. (19) & 4 & 25 \\
\hline Caliscelidae & & & sp. (12) & 5 & 2 \\
\hline Flatidae & Flatinae & Phantiini & Falcophantis westcotti (11) & 6 & 33 \\
\hline Flatidae & Flatinae & Siphantini & Siphanta patruelis (10) & 7 & 23 \\
\hline Tropiduchidae & Tropiduchinae & Gaetuliini & Alleloplasis sp. (13) & 8 & 3 \\
\hline Tropiduchidae & Tropiduchinae & Gaetuliini & Alleloplasis sp. (14) & 9 & 6 \\
\hline Tropiduchidae & Tropiduchinae & Tropiduchini & Oligaethus sp. (15) & 10 & 3 \\
\hline Issidae & & & sp. (9) & 11 and 12 & 2 \\
\hline Cixiidae & Cixiinae & & Dysoliarus unicornis (7) & 13 & 1 \\
\hline Cicadellidae & Ulopinae & Ulopini & ?Kahavalu sp. (42) & 14 & 1 \\
\hline Eurybrachidae & Platybrachinae & & sp. (8) & 15 & 6 \\
\hline Meenoplidae & Nisiinae & & Phaconeura sp. (5) & 16 & 3 \\
\hline Meenoplidae & Nisiinae & & Phaconeura sp. (6) & 17 & 33 \\
\hline Delphacidae & Delphacinae & Delphacini & sp. (1) & 18 & 3 \\
\hline Delphacidae & Delphacinae & Delphacini & Toya sp. (2) & 19 & 9 \\
\hline Delphacidae & Delphacinae & Delphacini & Sardia rostrata (3) & 20 & 1 \\
\hline Delphacidae & Delphacinae & Delphacini & sp. (4) & 21 & 18 \\
\hline Cicadellidae & Typhlocybinae & Empoascini & Austroasca histrionicula (56) & 22 & 5 \\
\hline Cicadellidae & Typhlocybinae & Empoascini & Austroasca viridigrisea (55) & 23 & 4 \\
\hline Membracidae & Centrotinae & Terentiini & Rigula sp. (72) & 24 & 3 \\
\hline Cicadellidae & Iassinae & Iassini & Batracomorphus adventitiosus (21) & 25 & 11 \\
\hline Cicadellidae & Iassinae & Iassini & Batracomorphus angustatus (20) & 26 & 6 \\
\hline Cicadellidae & Megophthalminae & Agalliini & Austroagallia torrida (68) & 27 & 2 \\
\hline Cicadellidae & Typhlocybinae & Erythroneurini & Anzygina sp. (59) & 28 & 16 \\
\hline Cicadellidae & Typhlocybinae & Erythroneurini & Empoascanara n.spZ06 (60) & 29 & 2 \\
\hline Cicadellidae & Typhlocybinae & Erythroneurini & Empoascanara peregrina $(61)$ & 30 & 16 \\
\hline Cicadellidae & Typhlocybinae & Erythroneurini & New genus ZA, sp.02A (69) & 31 & 7 \\
\hline Cicadellidae & Typhlocybinae & Erythroneurini & New genus ZA, sp.02 (62) & 32 & 15 \\
\hline Cicadellidae & Typhlocybinae & Erythroneurini & Anzygina n.sp.23A (65) & 33 & 14 \\
\hline Cicadellidae & Ulopinae & Cephalelini & Linacephalus foveolatus (58) & 34 & 3 \\
\hline Cicadellidae & Idiocerinae & & Pascoepus viridiceps (25) & 35 & 23 \\
\hline
\end{tabular}




\begin{tabular}{|c|c|c|c|c|c|}
\hline Family & Subfamily & Tribe & Morphospecies & $\begin{array}{l}\text { Genetic } \\
\text { species }\end{array}$ & $\mathrm{N}$ \\
\hline Cicadellidae & Deltocephalinae & Deltocephalini & sp. (46) & 36 & 22 \\
\hline Cicadellidae & Deltocephalinae & Deltocephalini & sp. (47) & 37 & 1 \\
\hline Cicadellidae & Deltocephalinae & Stenometopiini & sp. $(71)$ & 38 & 1 \\
\hline Cicadellidae & Deltocephalinae & Eupelicini & sp. (75) & 39 & 25 \\
\hline Cicadellidae & Deltocephalinae & Eupelicini & sp. (74) & 40 & 1 \\
\hline Cicadellidae & Deltocephalinae & Eupelicini & sp. (76) & 41 & 4 \\
\hline Cicadellidae & Deltocephalinae & Eupelicini & Mapochiella sp. (77) & 42 & 8 \\
\hline Cicadellidae & Deltocephalinae & Athysanini & Limotettix incertus (73) & 43 & 5 \\
\hline Cicadellidae & Tartessinae & & Protartessus spinosus (22) & 44 & 29 \\
\hline Cicadellidae & Tartessinae & & Newmaniana sp. (23) & 45 & 2 \\
\hline Cicadellidae & Idiocerinae & & Zaletta webbi (24) & 46 & 5 \\
\hline Cicadellidae & Eurymelinae & Ipoini & Ipoides hackeri (26) & 47 & 22 \\
\hline Cicadellidae & Deltocephalinae & Stenometopiini & Stirellus sp. (70) & 48 & 12 \\
\hline Cicadellidae & Deltocephalinae & Macrostelini & Balclutha incisa (53) & 49 & 3 \\
\hline Cicadellidae & Deltocephalinae & Macrostelini & Balclutha rosea (54) & 50 & 5 \\
\hline Cicadellidae & Deltocephalinae & Opsiini & Hishimonus sp. (63) & 51 & 1 \\
\hline Cicadellidae & Deltocephalinae & Opsiini & Goniagnathus sp. (52) & 52 & 1 \\
\hline Cicadellidae & Deltocephalinae & Athysanini & Exitianus nanus (31) & 53 & 8 \\
\hline Cicadellidae & Deltocephalinae & Macrostelini & Nesoclutha sp. (28) & 54 & 3 \\
\hline Cicadellidae & Deltocephalinae & Athysanini & Exitianus plebeius (30) & 55 & 4 \\
\hline Cicadellidae & Deltocephalinae & Deltocephalini & Maiestas knighti (33) & 56 & 14 \\
\hline Cicadellidae & Deltocephalinae & Deltocephalini & Maiestas sp. (32) & 57 & 3 \\
\hline Cicadellidae & Deltocephalinae & Opsiini & Orosius sp. (37) & 58 & 2 \\
\hline Cicadellidae & Deltocephalinae & Athysanini & Arawa sp. (36) & 59 & 1 \\
\hline Cicadellidae & Deltocephalinae & Deltocephalini & Horouta austrina (49) & 60 & 1 \\
\hline Cicadellidae & Deltocephalinae & Paralimnini & Soractellus sp. (50) & 61 & 1 \\
\hline Cicadellidae & Deltocephalinae & Deltocephalini & Maiestas sp. (51) & 62 & 1 \\
\hline Cicadellidae & Deltocephalinae & Deltocephalini & Horouta sp. (48) & 63 & 2 \\
\hline Cicadellidae & Deltocephalinae & Hecalini & Hecalus australis (27) & 64 & 1 \\
\hline Cicadellidae & Deltocephalinae & Paralimnini & Mayawa sp. (35) & 65 & 1 \\
\hline Cicadellidae & Deltocephalinae & Paralimnini & Mayawa sp. (34) & 66 & 1 \\
\hline Cicadellidae & Deltocephalinae & Athysanini & sp. (29) & 67 & 3 \\
\hline Cicadellidae & Deltocephalinae & Opsiini & Orosius sp. (41) & 68 & 1 \\
\hline Cicadellidae & Deltocephalinae & Opsiini & Orosius sp. (40) & 69 & 14 \\
\hline Cicadellidae & Deltocephalinae & Opsiini & Orosius sp. (39) & 70 & 2 \\
\hline Cicadellidae & Deltocephalinae & Opsiini & Orosius canberrensis (38) & 71 & 1 \\
\hline Cicadellidae & Deltocephalinae & Opsiini & Orosius argentatus (43) & 72 and 73 & 4 \\
\hline Cicadellidae & Deltocephalinae & Opsiini & Orosius sp. (44) & 74 & 1 \\
\hline Cicadellidae & Deltocephalinae & Opsiini & Orosius orientalis (45) & 75 and 76 & 14 \\
\hline
\end{tabular}


A)

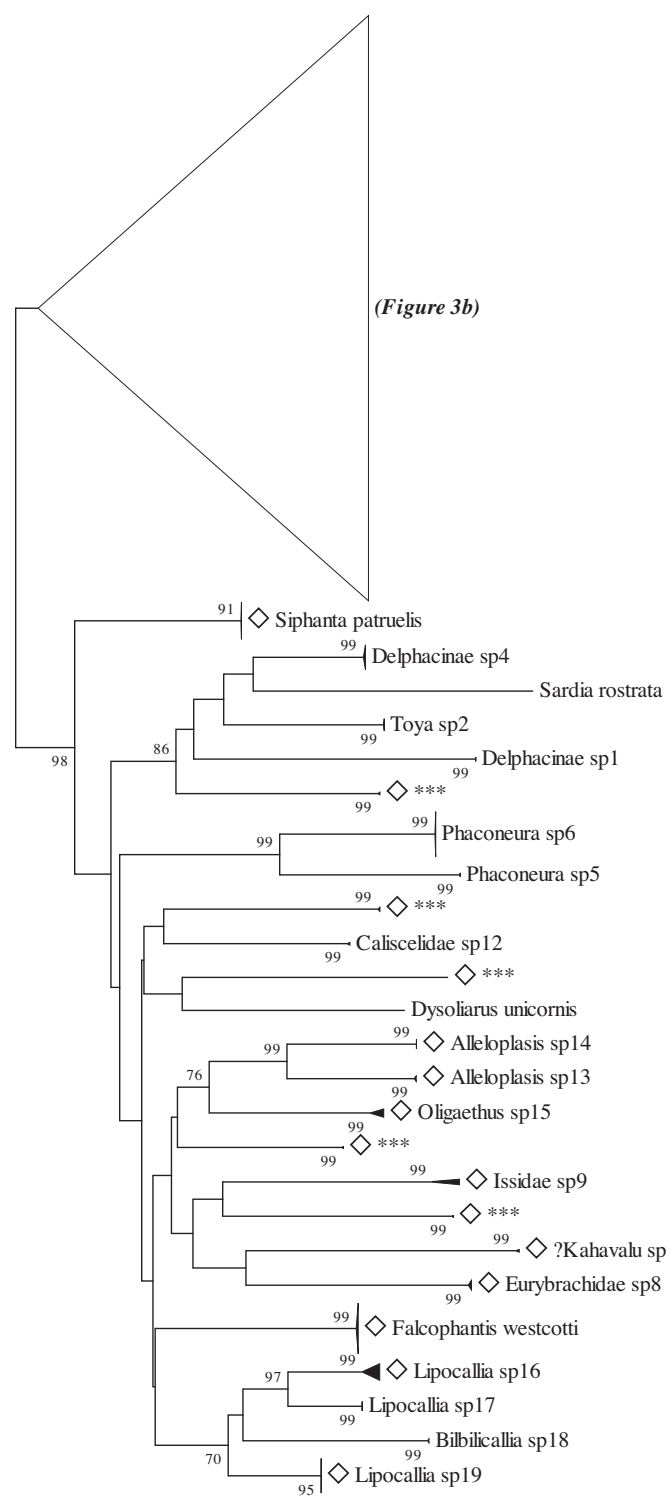

0.05
B)

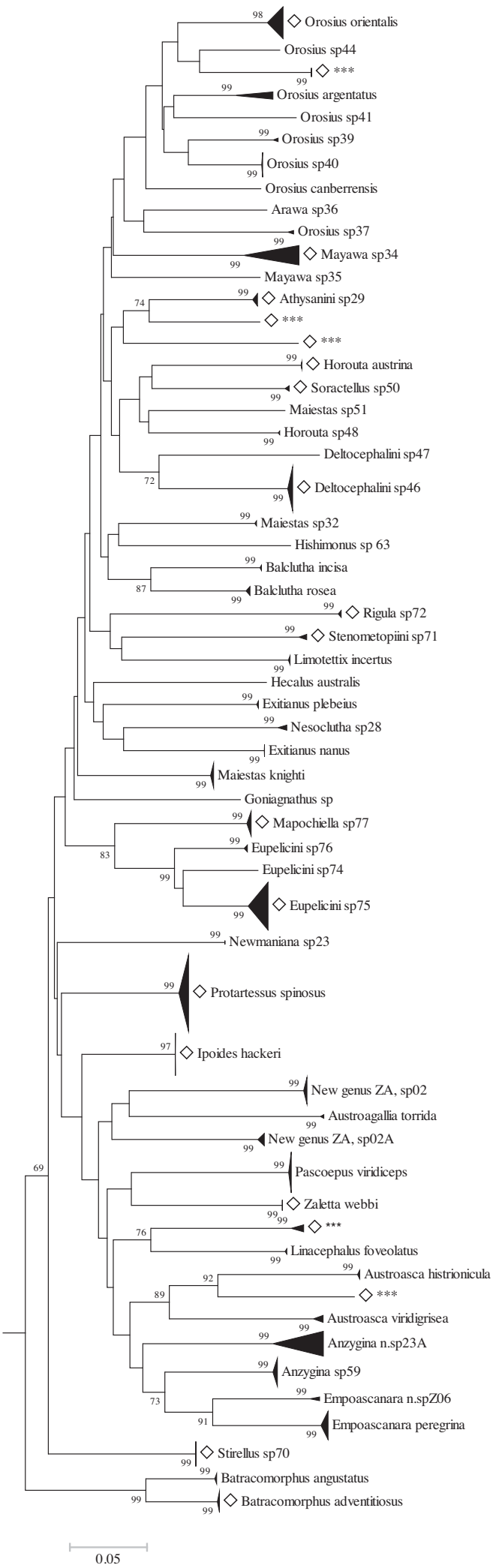

FIGURE $3 \quad$ Neighbour joining tree of all adult Auchenorrhyncha specimens $(N=546)$ and nymphs $(N=104)$; tree split as Figure 3a and 3b. Tree includes all specimens with DNA barcodes (> $500 \mathrm{bp}$ ) and partial barcodes ( $<500$ bp of sequence). Morphospecies labelled as per Table 2; presence of nymph(s) in species clade indicated by open diamond. Tips labelled *** indicate clade populated by nymphs only. Scale bar indicates percentage sequence difference adjusted by the Kimura-2 parameter model of evolution. Node support values $\geq 70 \%$ estimated by 1000 bootstrap replicates as indicated. 


\section{NYMPH DNA BARCODING}

DNA barcodes were retrieved from 106 nymphs (Appendix 2). Genetic relationships between adult and nymph specimens are seen in Figure 3. In total, 80 nymphs were identified to 24 morphospecies. The remaining 26 unidentified nymphs resolved in the tree as 10 terminal clades that were well supported $(>95 \%)$ and genetically distant from clades associated with adult morphospecies. Maximum genetic differences within these ten novel clades ranged from 0.16 to $1.63 \%$, and minimum distances to nearest sister clades ranged from 8.63 to $16.56 \%$.

\section{DISCUSSION}

Here we report the first instance of a survey of Auchenorrhyncha species present on Barrow Island, Western Australia, assessed using a combined morphological and molecular approach for species identification. We also examined the proposition that DNA barcoding used in conjunction with coalescent based sequence analyses can effectively complement traditional morphology based biodiversity assessment. Our results are largely supportive of this proposition and also demonstrate added benefits of using DNA barcoding in biodiversity analysis, particularly in circumstances where morphological criteria for species delimitation cannot be applied.

Key to this discussion was our principal result that genetic species delimitations identified among Auchenorrhyncha specimens using a single threshold GMYC model were highly congruent with our morphospecies identifications. Both approaches to species delimitation identified a minimum of 73 species present in the survey with total agreement among approaches regarding sample affiliation within delimited species. The $95 \%$ confidence interval at the single threshold GMYC model allowed recognition of an additional three putative species, where three morphospecies were each bifurcated as two separate genetic species. The maximum genetic difference observed in the three morphospecies was $4.66 \%$ in Orosius argentatus, differences in the other two split morphospecies ranged from $2.4-2.8 \%$. Sequence differences greater than $3 \%$ among faunal conspecifics at $\mathrm{CO} 1$ are infrequently observed in empirical population genetic and DNA barcoding surveys (Ferguson 2002; Hebert et al. 2003; Hebert et al. 2004a). Methods of species identification based on sequence distance statistics, such as the DNA barcode gap (Hebert et al. 2003; Hebert et al. 2004b) and other similar approaches (Ferri et al. 2009), are frequently used to derive operational standards for species delimitation. Distance based approaches are also frequently used in DNA barcoding studies to signify potential presence of cryptic diversity in morphologically cohesive species when limits are exceeded (Léfebure et al. 2006). These distance based approaches to species delimitation are computationally simple, are based directly on the empirical DNA barcode data and are seemingly applicable across a broad variety of fauna (Hebert et al. 2004b), albeit usually tailored for the focal study taxa. However these approaches ignore issues of genetic paraphyly (Trewick 2008) when single locus gene trees are not in accord with species trees due to a variety of biological, demographic, geographic or temporal processes (Doyle 1997; Avise 2000). Deep genetic divisions observed at a single locus within a morphospecies may equally signal presence of ancestral lineage retention or interspecies introgression as plausible alternatives to sympatry or parapatry of morphologically cryptic species (Funk and Omland 2003). Sequence differences exceeding $3 \%$ (or other similar empirically derived thresholds) in some reproductively cohesive insect species are likely to be more evident as the scale of both geographic sampling and sample replication is increased in DNA barcode surveys (Trewick 2008; Bergstein et al. 2012; but see Lukhtanov et al. 2009). Increasingly there are calls to the DNA barcode community to treat DNA barcode species identifications as hypotheses to be tested in an integrative taxonomic framework, incorporating multiple independent data (behavioural, ecological, molecular, morphological) to examine the cohesiveness of species boundaries defining examined taxa (Dayrat 2005; DeSalle et al. 2005; Padial et al. 2010; SchlickSteiner et al. 2010; Goldstein and DeSalle 2011). In this current study, our subsequent morphological re-examination of specimens at each of the three genetically split morphospecies failed to identify any corroborative morphological evidence supportive of these genetic species divisions. We conservatively argue there is insufficient evidence in the current sample to determine if the three instances of genetic splitting detected here result from co-existence of morphologically cryptic species, retention of divergent mitochondrial lineages in the population of the species, or other causes such as cross species introgression. Future work to examine independent nuclear sequence diversity (or other independent comparative data types) across a broader geographic sampling area of the three morphospecies is needed to test these competing hypotheses.

In contrast to that seen in the single threshold GMYC model, species delimitations using the multiple threshold GMYC were incongruent for $\sim 60 \%$ of the morphospecies identifications, with differences apparent due to both fusing and splitting of the morphospecies (Sup.Table 1). For example, the multiple threshold GMYC model 
fused 30 distinct morphological species into 12 putative species. In many of those instances, morphospecies identified by a single haplotype were lumped with genetically diverse sister species or genera and, in a few instances, species from different subfamilies were fused together (refer Sup. Table 1). Average maximum sequence difference within these fused genetic species was $>17 \%$, far exceeding empirically observed levels of intraspecific divergence at mitochondrial CO1 in eukaryotes (Hebert et al. 2003). The multiple threshold model also split 16 morphospecies into two or more genetic species. In several instances, haplotypes which minimally differed by $<1 \%$ sequence difference in a morphospecies were delimited as separate genetic species. Clearly in the current study, the single threshold GMYC model provided greater congruence to the morphological analyses than did the multiple threshold model, both with the number of species delimited and with specimen affiliation within the delimited species. This is surprising, given that the multiple threshold GMYC model was a significantly better fit to the genetic data compared to the single threshold model $\left(\log \mathrm{L}_{\text {multiple }}=2747.4\right.$ vs $\log \mathrm{L}_{\text {single }}=$ $2640.2,{ }^{2}=214.41$, d.f., $=21, \mathrm{P}<0.001$ ), a result which indicated species boundaries varied significantly among genetic lineages in the sample phylogeny. A contrasting outcome was observed by Monaghan et al. (2009) in their GMYC analyses of four insect orders sampled from Madagascar. In that study, the multiple threshold model resulted in a slight overall trend in morphospecies fusing but fewer instances of splitting and greater congruence to the morphospecies delimitations than did the single threshold model. Evidence in this current study suggests that acceptance of species delimitations of the multiple over the single threshold model, based solely on comparison of likelihood scores between these two models, can lead to erroneous estimations of species limits in a purely genetic survey. Reasons why the multiple threshold model performed poorly in this current study are unclear. However there were several instances using the multiple threshold model, where morphospecies represented by single haplotypes were merged with distantly related and genetically heterogeneous morphospecies to form single putative species. The circumstances leading to this outcome may be related to the effect of high variance in average effective population sizes relative to species divergence times in the gene tree (Fujisawa and Barraclough 2013 in press). Accuracy of the multiple model to optimise multiple diversification events may be affected when there is an abundance of species represented by singleton haplotypes that are genetically closest to more heterogeneous taxa. Greater scrutiny of single and multiple threshold GMYC models outcomes using a variety of simulated species diversification and population coalescent events may provide some insight into circumstances where the outcomes of the two models are likely to differ and or provide erroneous species delimitations. Very recent simulation work reported by Fujisawa and Barraclough (2013, in press) indicates the multiple threshold model is marginally less conservative than the single threshold model in regards to false positive error rates, and has greater incidence of species splitting across a variety of demographic scenarios. Species splitting was most apparent in simulations using an abundance of recently declining species populations, where excess recent coalescent events resulted in a greater prevalence of artefactual clusters detected by the multiple threshold model. The caveat from these simulations, predictions and our empirical analysis, is that modelling multiple transitions from speciation to coalescence branching patterns may erroneously estimate species numbers, in some circumstances delimiting species complexes as opposed to individual species (Pons et al. 2006), and in other cases resulting in splitting of species (Fujisawa and Barraclough 2013 in press).

The final inventory of 73 Auchenorrhyncha species at Barrow Island congruently delimited by morphology and genetics includes species from nine of the fifteen Fulgoroid (planthopper) families, and the two Membracoid (leaf/treehopper and horned treehopper) families present in Australia (Fletcher 2009). Cicadellidae are well represented (Table 2; Figure 2) and include eight of the seventeen leaf/treehopper subfamilies present in Australia. Species allocation in the inventory is dominated by leafhoppers ( $>72 \%$ of all detected species), most of which are Deltocephalinae species ( $>48 \%$ of all detected species). Cosmopolitan species account for at least $36 \%$ of those detected at Barrow Island, but this is likely to be under-representative of the actual percentage of cosmopolitans present. One of the unresolved issues remaining from this survey, concerns the identity of indeterminate species delimited by the morphological and molecular approaches used here. In the case of adult specimens, $64 \%$ of taxa are taxonomically unresolved at the species level. These included a single morphospecies unable to be identified by morphology due to specimen condition (refer Table 2, Issidae, species (9)), and 46 putative species that either lacked male specimens for taxonomic identification, or could not be identified to formally recognised species present in Australia. All unresolved specimen sequences were queried for genetic similarity to sequence records and accessions in BOLD and GenBank [last accessed 20.10. 2012], but failed to be identified at $97 \%$ similarity. Closest genetic matches (data not shown) were to various Auchenorrhyncha accessions, 
suggesting the diversity of unidentified specimens in this study was not inflated by presence of contaminations or non-target taxa. These specimens must necessarily remain as interim undescribed taxa until matching DNA barcodes from physically complete and taxonomically identified adult male specimens are available. Regardless, permanent DNA barcode sequence records of all interim and indeterminate species detected here are stored in BOLD and are available for future sequence enquires and taxonomic annotations.

One of the major advantages of using DNA barcoding as an investigative tool for biodiversity analyses, is its unique facility to provide species identifications for specimens that are either physically degraded (deWaard et al. 2010), or of life stages with taxonomically intractable morphology (Hebert et al. 2004). Typically, these efforts rely on a pre-existing library of DNA barcodes from taxonomically described species, which can be used as references in distance based comparisons against a query specimen. In this study, nymphs were DNA barcoded and either resolved as conspecifics to adult morphospecies (80 nymphs identified to 24 adult morphospecies; Figure 3) or unresolved (26 nymphs grouped as ten novel monophyletic clades) with no match to adult specimens or sequence records at BOLD or GenBank [last accessed 20.10. 2012]. This is a similar outcome to that reported by Ahrens et al. (2007) for chafers (Coleoptera: Scarabaeidae) where $21 \%$ of genetically delimited species encountered as morphologically indistinct larvae could not be genetically associated to co-sampled adult specimens. In our study, the minimum genetic distances to these novel nymph clades was $>8.6 \%$, and marginally greater than the minimum nearest neighbour distances among the adult species clades (> 7.2\%); it remains to be determined if this novel diversity present among the nymphs is representative of undetected adult taxa (as inferred by the genetic distance data), or is representative of novel and deep population genetic variation within identified taxa. Regardless, this novel diversity would have remained undetected if specimen identifications were based solely on morphology. Taxonomic keys available for the Auchenorrhyncha describe and identify species based primarily on adult male specimens; keys for identification of their nymphs are either unavailable, or at best, limited to the higher levels of classification (e.g. Dmitriev 2009). As a result, morphological surveys of Auchenorrhyncha may underestimate species diversity if a portion of immature specimens present at a location are not conspecific with any sampled adults. A similar outcome may result from excessive presence of physically degraded specimens. The extent of this "hidden" diversity will vary among sampling efforts according to both the sampling methods employed for specimen collection and the seasonality of adult / immature specimen occurrence at sample locations. To this end, inclusion of DNA barcoding in future Auchenorrhyncha sampling efforts will provide a means not only to identify presence of "hidden" diversity, but also provide ecological information concerning the seasonality of occurrence among life stages of particular species (Ahrens et al. 2007). For these reasons, DNA barcoding could effectively inform the design and duration of ongoing survey efforts, particularly if a predetermined threshold of hidden or seasonal diversity was exceeded in pilot analyses.

\section{CONCLUSIONS}

The high level of congruence in species delimitation observed here, between independent examinations of morphology and DNA barcodes, provides some confidence that future DNA barcode assays of Auchenorrhyncha are likely to provide species identifications and delimitations at this group with a high level of confidence. Furthermore the approach is applicable and informative across a range of Auchenorrhyncha specimen types and life stages, and therefore likely to be an invaluable investigative tool for surveying alpha taxonomy in this group. This view is tempered by the caveat demonstrated here, that an inappropriate choice of genetic model used for analysis of DNA barcodes can substantially over and under estimate species assignments. Therefore we are supportive of recent calls for DNA barcode species hypotheses to be holistically tested and combined with independent data types that are informative of species boundaries and can be compared in an integrative taxonomic framework.

\section{ACKNOWLEDGEMENTS}

Funding for the molecular work was provided by the NSW BioFirst Initiative grant to the NSW Agricultural Genomics Centre.

\section{REFERENCES}

Ahrens, D., Monaghan, M.T. and Vogler, A.P. (2007). DNA-based taxonomy for associating adults and larvae in multi-species assemblages of chafers (Coleoptera: Scarabaeidae). Molecular Phylogenetics and Evolution 44: 436-449.

Avise, J.C. (2000). Phylogeography: the History and Formation of Species. Harvard University Press, Cambridge, MA.

Bennett, G.M. and O'Grady, P.M. (2012). Host-plants drive insect diversity: Phylogeny, diversity, and origins of native Hawaiian leafhoppers (Cicadellidae: Nesophrosyne). Molecular Phylogenetics and Evolution 65: 705-717. 
Bergsten, J., Bilton, D.T., Fujisawa, T., Elliot, M., Monaghan, M.T., Balke, M., Hendrich, L., Geijer, J., Herrmann, J., Foster, G.N., Ribera, I., Nilsson, A.N., Barraclough, T.G. and Vogler A.P. (2012). The effect of geographical scale of sampling on DNA barcoding. Systematic Biology 61: 851-869.

Bluemel, J.K., King, R.A., Virant-Doberlet, M., and Symondson, W.O.C. (2011). Primers for identification of type and other archived specimens of Aphrodes leafhoppers (Hemiptera, Cicadellidae). Molecular Ecology Resources 11: 770-774.

Callan, S.K., Majer, J.D., Edwards, K. and Moro, D. (2011). Documenting the terrestrial invertebrate fauna of Barrow Island, Western Australia. Australian Journal of Entomology 50: 323-343.

Cho, S., Mitchell, A., Mitter, C., Regier, J., Matthews, M. and Robertson, R. (2008). Molecular phylogenetics of heliothine moths (Lepidoptera, Noctuidae, Heliothinae), with comments on the evolution of host range and pest status. Systematic Entomology 33: 581-594.

Cummings, M.P., Neel, M.C. and Shaw, K.L. (2008). A genealogical approach to quantifying lineage divergence. Evolution 62: 2411-2422.

Davis, J.I. and Nixon, K.C. (1992). Populations, genetic variation, and the delimitation of phylogenetic species. Systematic Biology 41: 421-435.

Dayrat, B. (2005). Towards integrative taxonomy. Biological Journal of the Linnean Society 85: 407-415.

DeSalle, R., Egan, M.G. and Siddal, M. (2005). The unholy trinity: taxonomy, species.

delimitation and DNA barcoding. Philosophical Transactions of the Royal Society B 360: 1905-1916.

deWaard, J.R., Mitchell, A., Keena, M.A., Gopurenko, D., Boykin, L.M., Armstrong, K.F., Pogue, M.G., Lima, J., Floyd, R., Hanner, R.H., Humble, L.M. (2010). Towards a global barcode library for Lymantria (Lepidoptera: Lymantriinae) tussock moths of biosecurity concern. Public Library of Science One 5: e14280.

Dmitriev, D.A. (2009). Nymphs of some Nearctic leafhoppers (Homoptera, Cicadellidae) with description of a new tribe. ZooKeys 29: 13-33.

Doyle, J.J. (1997). Trees within trees. Genes and species, molecules and morphology. Systematic Biology 46: 537-553.

Ferri, E., Barbuto, M., Bain, O., Galimberti, A., Uni, S., Guerrero, R., Ferté, H. Bandi, C., Martin, C. and Casiraghi M. (2009). Integrated taxonomy: traditional approach and DNA barcoding for the identification of filarioid worms and related parasites (Nematoda). Frontiers in Zoology 6: 1-12.

Fletcher, M.J. (2009). Identification keys and checklists for the leafhoppers, planthoppers and their relatives occurring in Australia and neighbouring areas (Hemiptera: Auchenorrhyncha). http://www1.dpi. nsw.gov.au/keys/leafhop/index.html

Ferguson, J.W.H. (2002). On the use of genetic divergence for identifying species. Biological Journal of the Linnean Society 75: 509-516.

Folmer, O., Black, M., Hoeh, W., Lutz, R. and Vrijenhoek, R. (1994). DNA primers for amplification of mitochondrial cytochrome c oxidase subunit I from diverse metazoan invertebrates. Molecular Marine Biology and Biotechnology 3: 294-299.

Fujisawa, T. and Barraclough T.G. (2013). Delimiting species using single-locus data and the generalized mixed yule coalescent (GMYC) approach: A revised method and evaluation on simulated datasets. Systematic Biology (early online release: syt033v1syt033).

Fujita, M.K., Leaché, A.D., Burbrink, F.T., McGuire, J.A. and Moritz, C. (2012). Coalescent-based species delimitation in an integrative taxonomy. Trends in Ecology and Evolution 27: 480-488.

Funk, D.J. and Omland, K.E. (2003). Species-level paraphyly and polyphyly: frequency, causes, and consequences, with insights from animal mitochondrial DNA. Annual Review of Ecology, Evolution and Systematics 34: 397-423.

Goldstein, P.Z. and DeSalle, R. (2011). Integrating DNA barcode data and taxonomic practice: Determination, discovery, and description. Bioessays 33: 135-147.

Hall, T.A. (1999). BioEdit: a user-friendly biological sequence alignment editor and analysis program for Windows 95/98/NT. Nucleic Acids Symposium Series 41: 95-98.

Hebert, P.D.N., Cywinska, A., Ball, S.L. and deWaard, J.R. (2003). Biological identifications through DNA barcodes. Proceedings of the Royal Society of London. Series B. Biological Sciences 270: 313-321.

Hebert, P.D.N., Penton, E.H., Burns, J.M., Janzen, D.H. and Hallwachs, W. (2004a). Ten species in one: DNA barcoding reveals cryptic species in the neotropical skipper butterfly Astraptes fulgerator. Proceedings of the National Academy of Sciences of the United States of America 101: 14812-14817.

Hebert, P.D.N., Stoeckle, M.Y., Zemlak, T.S. and Francis, C.M. (2004b). Identification of birds through DNA barcodes. Public Library of Science Biology 2: e312.

Jansen, G., Savolainen, R., and Vepsalainen, K. (2009). DNA barcoding as a heuristic tool for classifying undescribed Nearctic Myrmica ants (Hymenoptera: Formicidae). Zoologica Scripta 38: 527-536.

Kamitani, S. (2011). DNA Barcodes of Japanese Leafhoppers. Esakia: Occasional Papers of the Hikosan Biological Laboratory in Entomology 50: 81-88.

Kimura, M. (1980). A simple method for estimating evolutionary rate of base substitutions through comparative studies of nucleotide sequences. Journal of Molecular Evolution 16:111-120.

Leliaert, F., Verbruggen, H., Wysor, B. and De Clerck, O. (2009). DNA taxonomy in morphologically plastic taxa: algorithmic species delimitation in the Boodlea complex (Chlorophyta: Siphonocladales). Molecular Phylogenetics and Evolution 53: 122-133.

Le Roux, J.J. and Rubinoff, D. (2009). Molecular data reveals California as the potential source of an invasive leafhopper species, Macrosteles sp. nr. severini, transmitting the aster yellows phytoplasma in Hawaii. Annals of Applied Biology 154: 419-427.

Lefébure, T., Douady, C.J., Gouy, M. and Gibert, J. (2006). Relationship between

morphological taxonomy and molecular divergence within Crustacea: proposal of a molecular threshold 
to help species delimitation. Molecular Phylogenetics and Evolution 40: 435-447.

Lukhtanov, V.A., Sourakov, A., Zakharov, E.V. and Hebert P.D. (2009). DNA barcoding Central Asian butterflies: increasing geographical dimension does not significantly reduce the success of species identification. Molecular Ecology Resources 9: 13021310.

Marya ska-Nadachowska, A., Lachowska-Cierlik, D., Drosopoulos, S., Kajtoch, Ł. and Kuznetsova, V.G. (2010). Molecular phylogeny of the Mediterranean species of Philaenus (Hemiptera: Auchenorrhyncha: Aphrophoridae) using mitochondrial and nuclear DNA sequences. Systematic Entomology 35: 318-328.

Meier, R., Zhang, G.Y. and Ali, F. (2008). The use of mean instead of smallest interspecific distances exaggerates the size of the "barcoding gap" and leads to misidentification. Systematic Biology 57: 809-813.

Monaghan, M.T., Wild, R., Elliot, M., Fujisawa, T., Balke, M., Inward, D.J.G., Lees, D.C., Ranaivosolo, R., Eggleton, P., Barraclough, T.G. and Vogler, A.P. (2009). Accelerated species inventory on Madagascar using coalescent-based models of species delineation. Systematic Biology 58: 298-311.

Padial, J.M., Miralles, A., De la Riva I. and Vences M. (2010). The integrative future of taxonomy. Frontiers in Zoology 7: 16.

Park, D.S., Suh, S.J., Hebert, P.D., Oh, H.W. and Hong, K.J. (2011a). DNA barcodes for two scale insect families, mealybugs (Hemiptera: Pseudococcidae) and armored scales (Hemiptera: Diaspididae). Bulletin of Entomological Research 101: 429-434.

Park, D.S., Foottit, R., Maw, E. and Hebert, P.D.N. (2011b). Barcoding Bugs: DNA-Based Identification of the True Bugs (Insecta: Hemiptera: Heteroptera). Public Library of Science ONE 6: e18749.

Papadopoulou, A., Bergsten, J., Fujisawa, T., Monaghan, M.T., Barraclough, T.G. and Vogler, A.P. (2008). Speciation and DNA barcodes: testing the effects of dispersal on the formation of discrete sequence clusters. Philosophical Transactions of the Royal Society B. 363: 2987-2996.

Paradis, E., Claude, J. and Strimmer, K. (2004). APE: analyses of phylogenetics and evolution in $R$ language. Bioinformatics 20: 289-290.

Paradis, E. (2006). Analyses of Phylogenetics and Evolution with R. Springer, New York.

Pons, J., Barraclough, T.G., Gomez-Zurita, J., Cardoso, A., Duran, D.P., Hazell, S., Kamoun, S., Sumlin, W.D. and Vogler, A.P. (2006). Sequence-based species delimitation for the DNA taxonomy of undescribed insects. Systematic Biology 55: 595-609.
Posada, D. (2008). jModelTest: Phylogenetic Model Averaging. Molecular Biology and Evolution 25: 12531256.

Ratnasingham, S. and Hebert, P.D.N. (2007). BOLD: the barcode of life data system (http://www. barcodinglife.org). Molecular Ecology Notes 7: 355-364.

Rosenberg, N.A. (2007). Statistical tests for taxonomic distinctiveness from observations of monophyly. Evolution 61: 317-323.

Saitou, N. and Nei, M. (1987). The neighbor-joining method: A new method for reconstructing phylogenetic trees. Molecular Biology and Evolution 4: 406-425.

Schlick-Steiner, B.C., Steiner, F.M., Seifert, B., Stauffer, C., Christian, E. and Crozier, R.H. (2010). Integrative taxonomy: A multisource approach to exploring biodiversity. Annual Review of Entomology 55: 421-438.

Seabra, S.G., Pina-Martins, F., Marabuto, E., Yurtsever, S., Halkka, O., Quartau, J.A. and Paulo, O.S. (2010). Molecular phylogeny and DNA barcoding in the meadow-spittlebug Philaenus spumarius (Hemiptera, Cercopidae) and its related species. Molecular Phylogenetics and Evolution 56: 462-467.

Simon, C., Frati, F., Beckenbach, A., Crespi, B., Liu, H. and Flook, P. (1994). Evolution, weighting and phylogenetic utility of mitochondrial gene-sequences and compilation of conserved polymerase chain reaction primers. Annals of the Entomological Society of America 87: 651-701.

Swofford, D.L. (1998). PAUP*. Phylogenetic Analysis Using Parsimony (*and other Methods). Version 4.0b10. Sinauer Associates, Sunderland, Massachusetts.

Tamura, K., Dudley, J., Nei, M. and Kumar, S. (2007). MEGA4: Molecular Evolutionary Genetics Analysis (MEGA) software version 4.0. Molecular Biology and Evolution 24:1596-1599.

Trewick, S.A. (2008). DNA Barcoding is not enough: mismatch of taxonomy and genealogy in New Zealand grasshoppers (Orthoptera: Acrididae). Cladistics 24: 240-254.

Villesen, P. (2007). FaBox: an online toolbox for fasta sequences. Molecular Ecology Notes 7: 965-968.

Will, K.W. and Rubinoff, D. (2004). Myth on the molecule: DNA barcodes for species cannot replace morphology for identification and classification. Cladistics 20: 47-55.

Yang, Z. and Rannala, B. (2010). Bayesian species delimitation using multilocus sequence data. Proceedings of the National Academy of Sciences of the United States of America 107: 9264-9269.

MANUSCRIPT RECEIVED 30 NOVEMBER 2012; ACCEPTED 12 JULY 2013. 


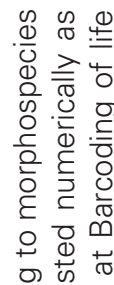

응

흥 $\frac{\bar{d}}{\frac{0}{0}} \frac{0}{\pi}$

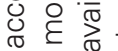

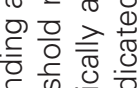

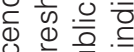

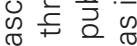

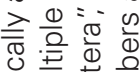

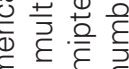

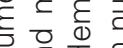

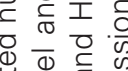

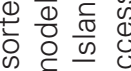

की

टे

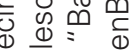

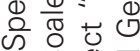

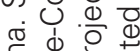

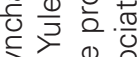

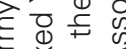

政

远

文牙

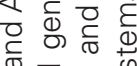

흠응 웡 को

उत्

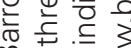

$\pm \frac{0}{\pi} \approx$

可

응응

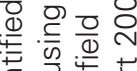

ब

구유

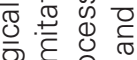

응 흥 응

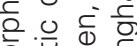

这

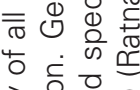

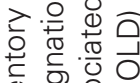

它.

要要这

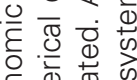

०े क्ष के

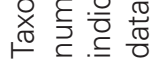

$\underset{\frac{x}{2}}{\frac{\bar{x}}{2}}$

ब客

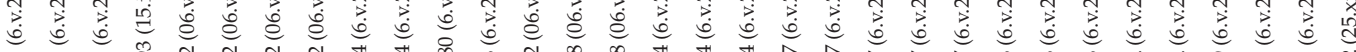

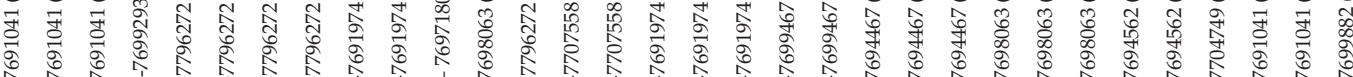

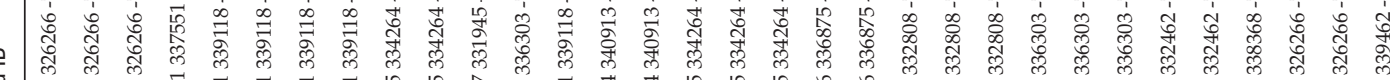

음

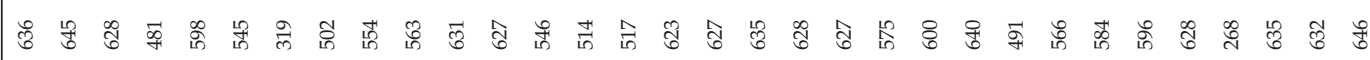

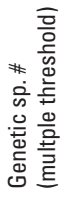

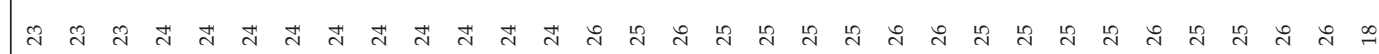

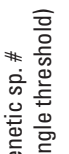

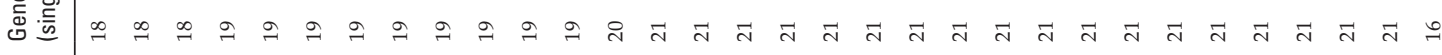

을

œ

山

山 $\Sigma$

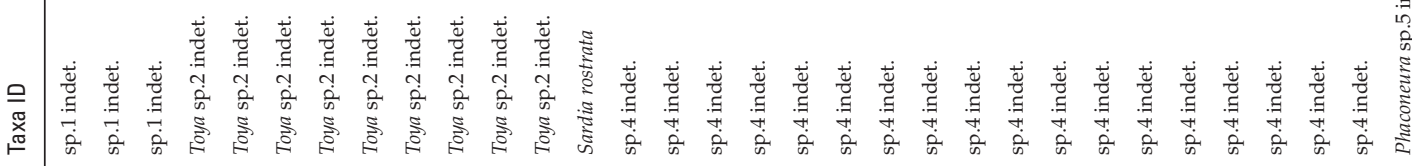

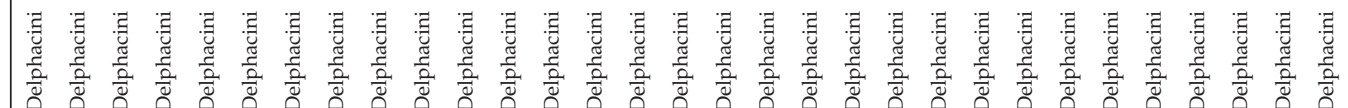

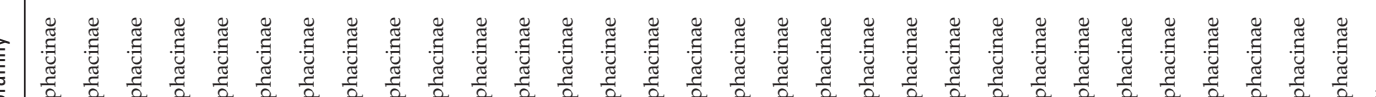

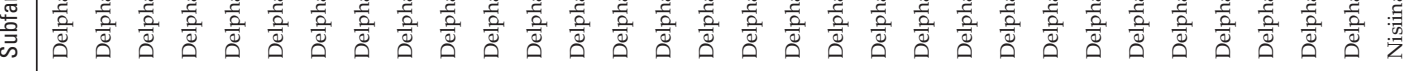

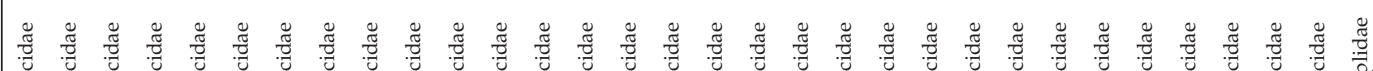

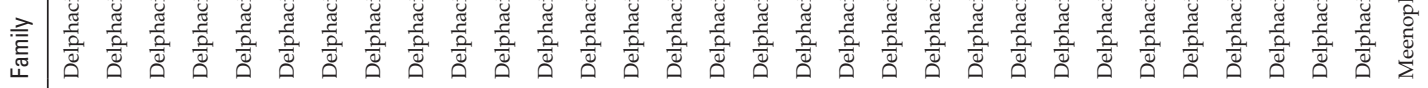

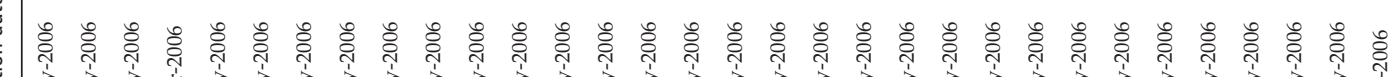

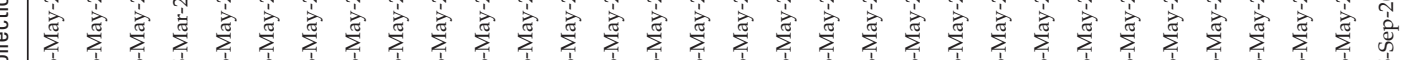

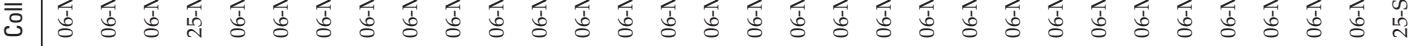

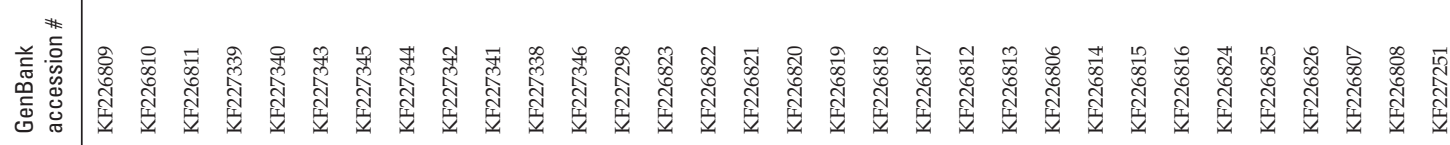

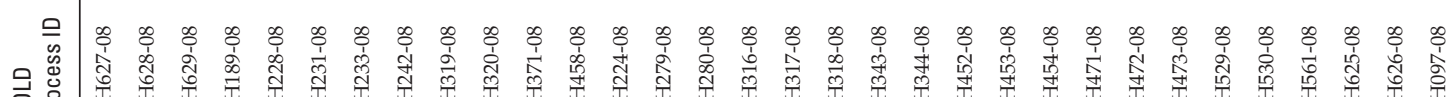

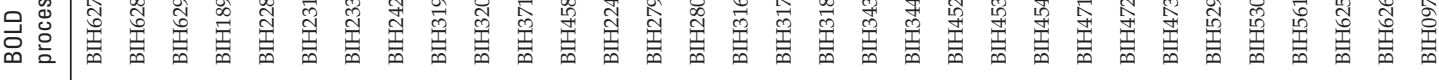

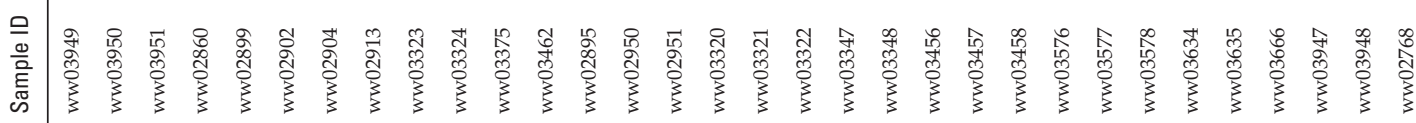




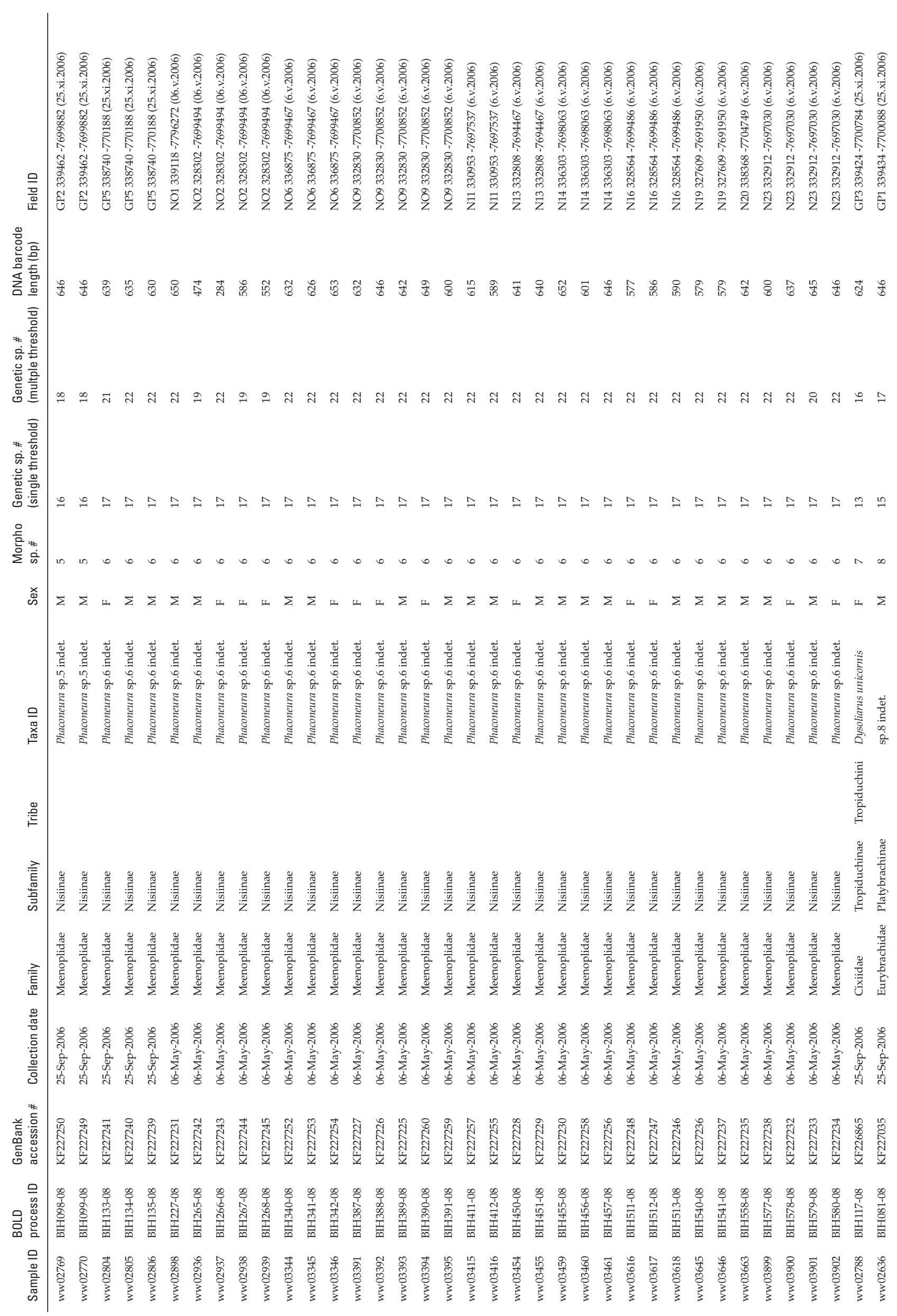




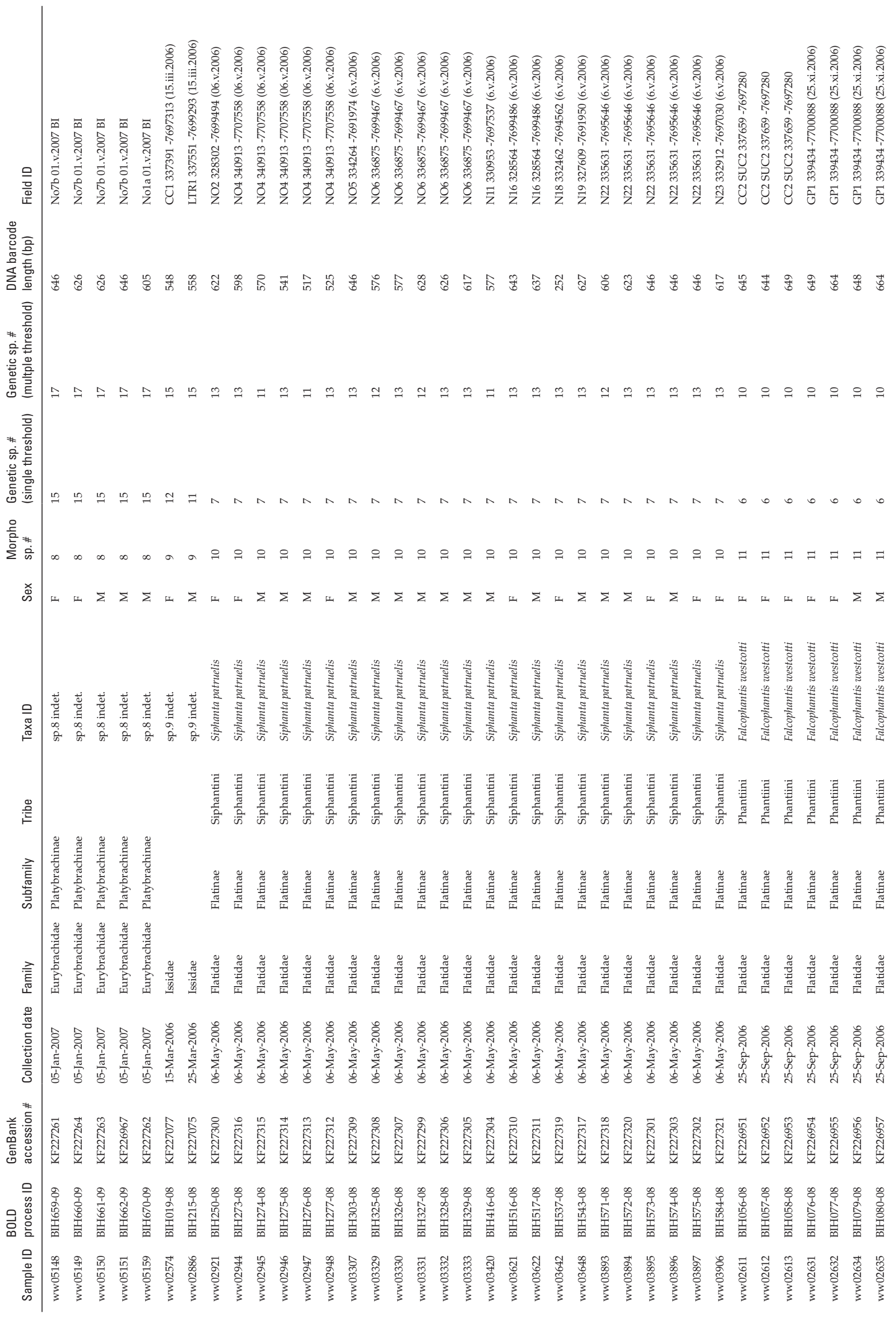


言

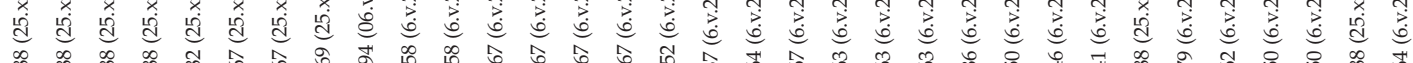

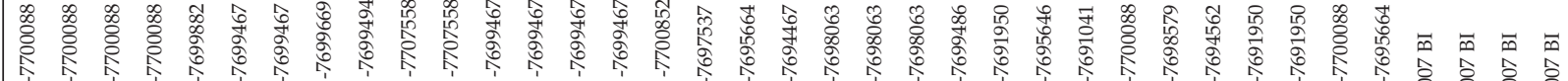

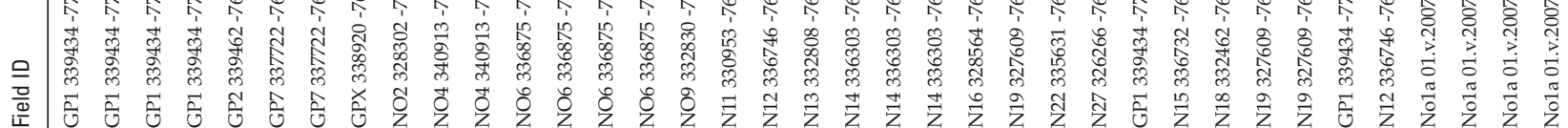

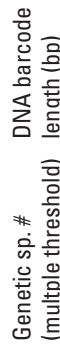

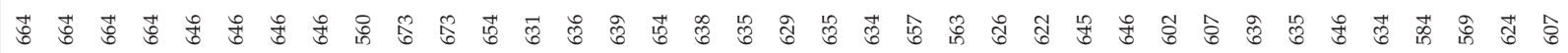

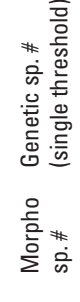

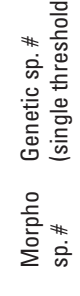

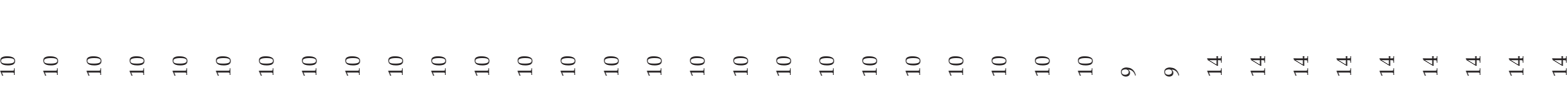

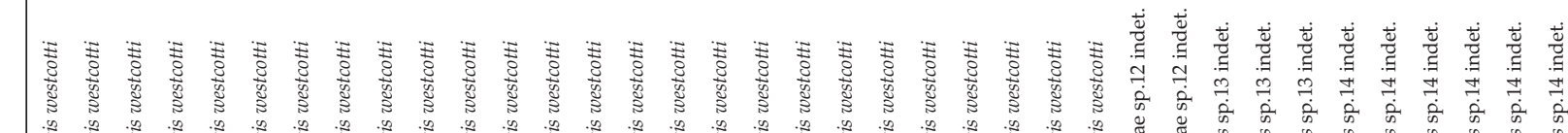

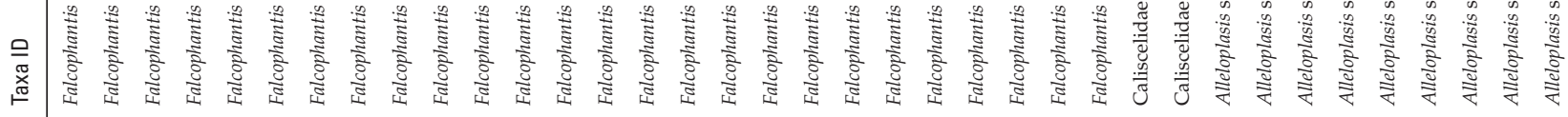

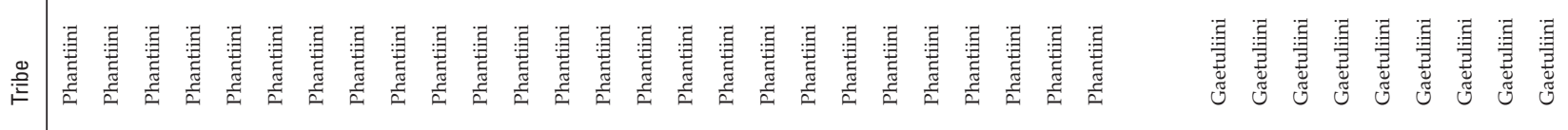
mmmmmmm

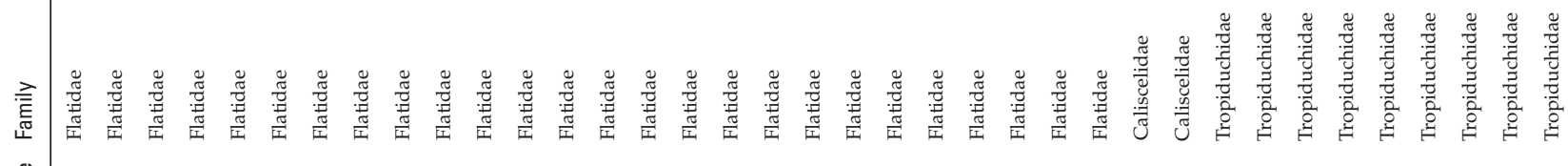

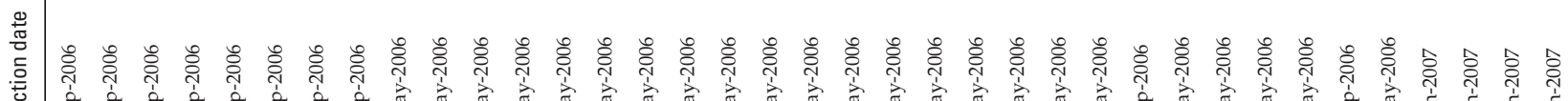

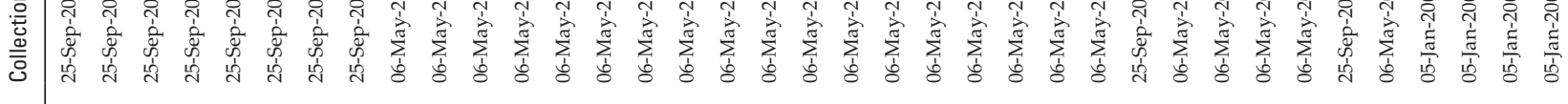

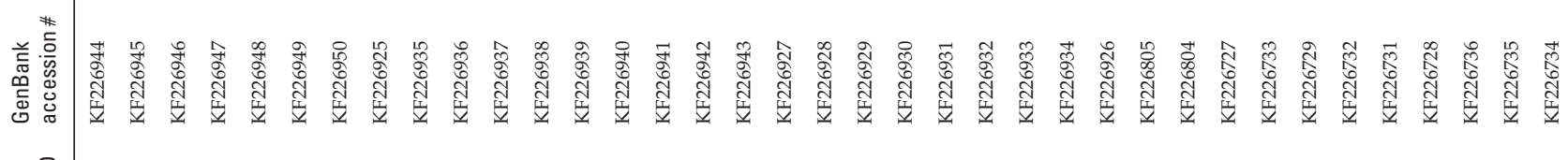

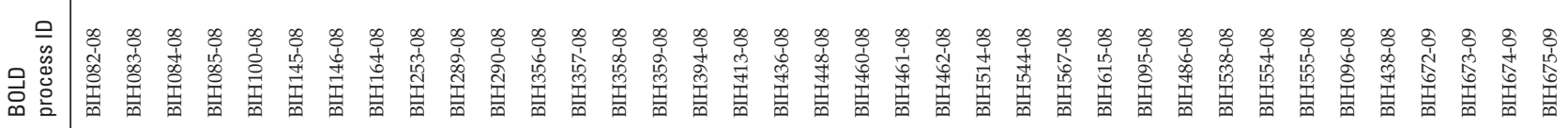

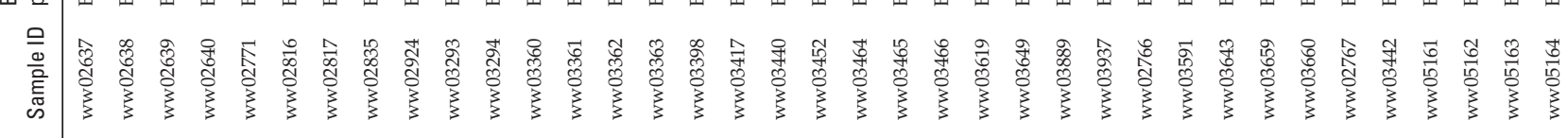




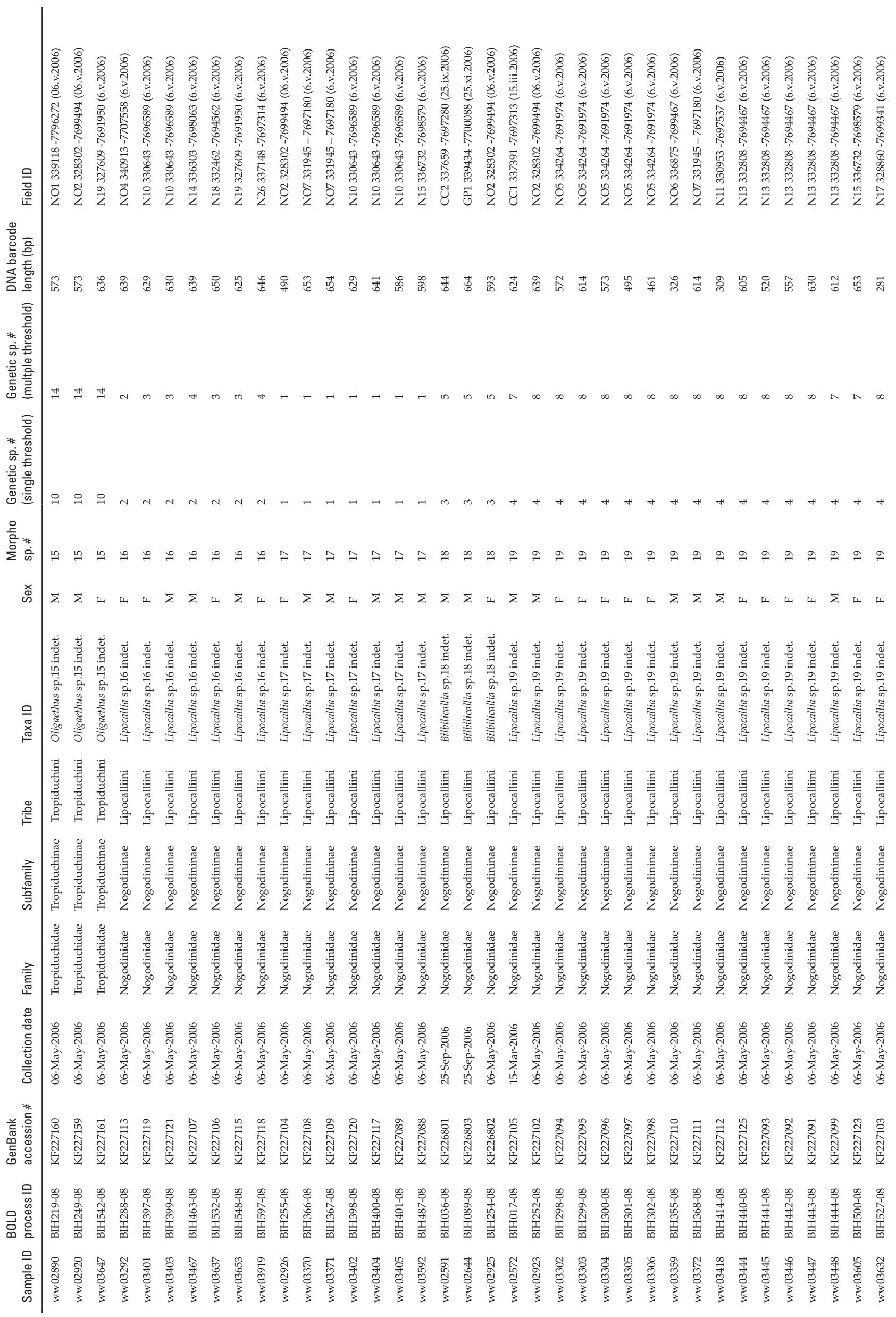




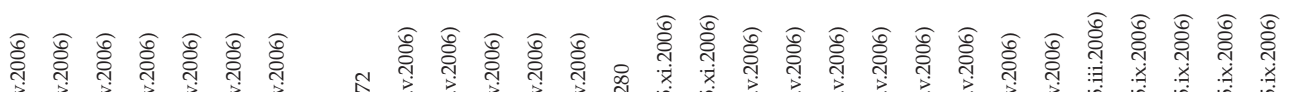

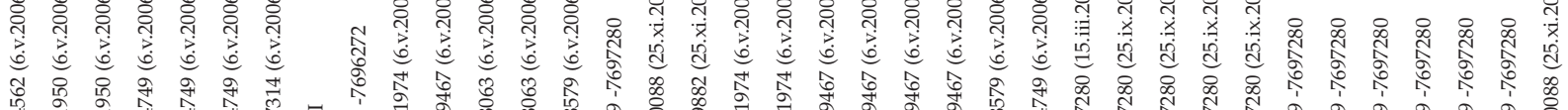

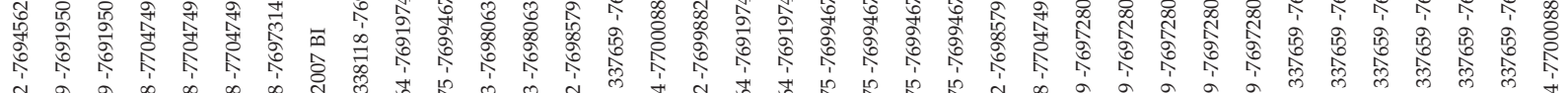

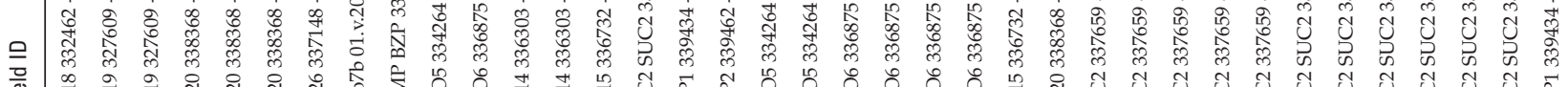

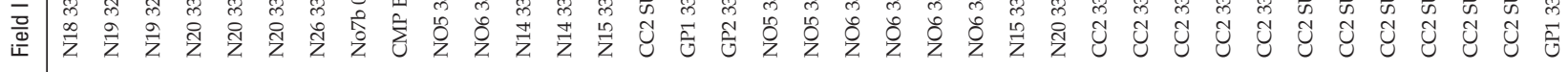

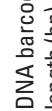

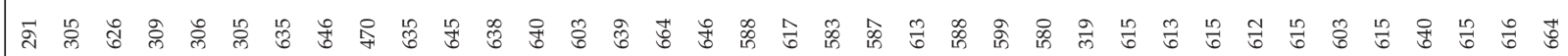

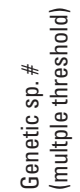

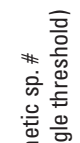

曾

을

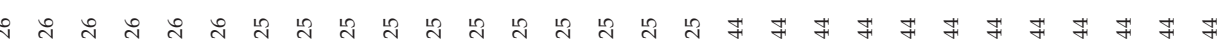
$\stackrel{\times}{\infty}$

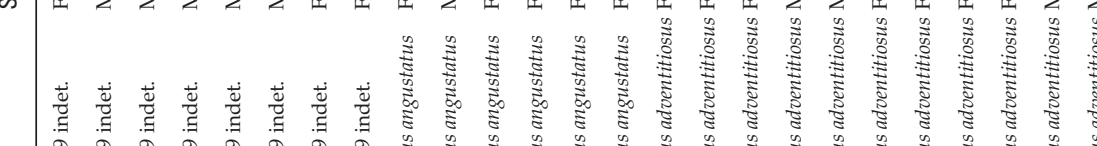

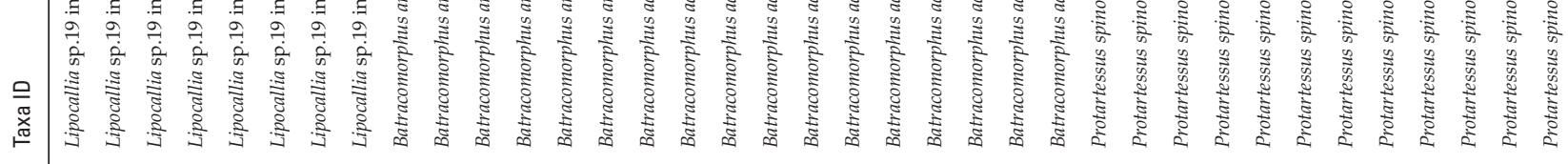

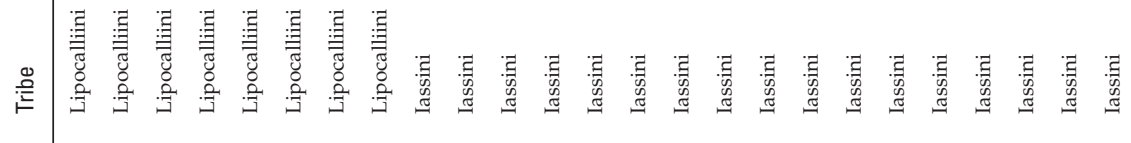

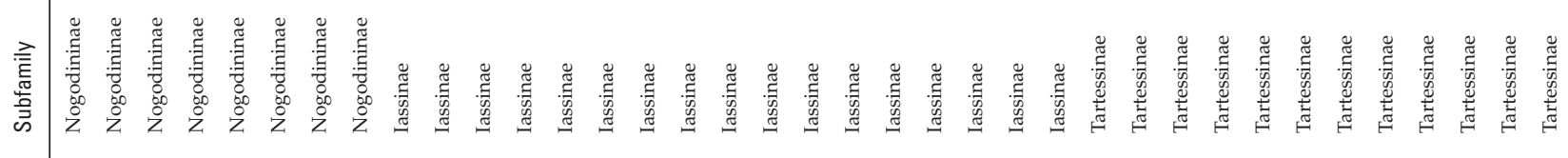

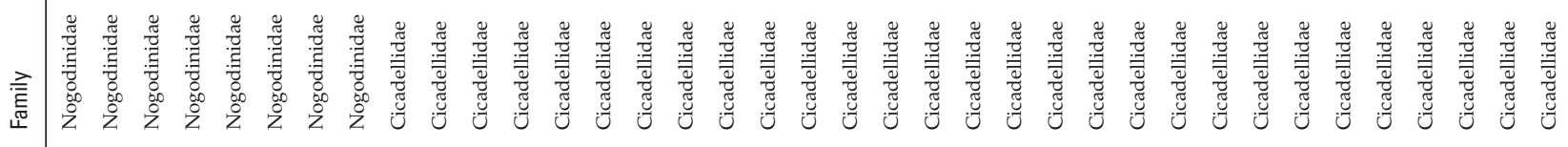

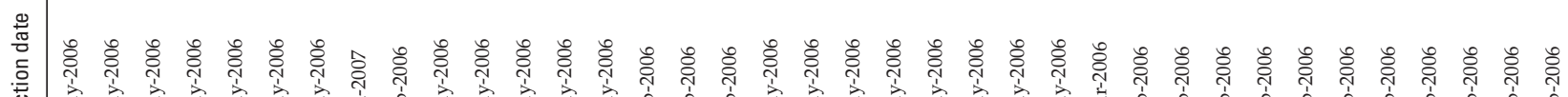

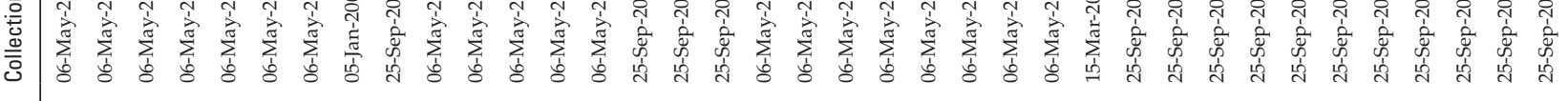

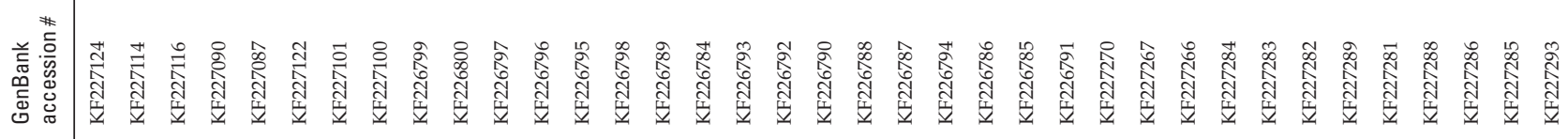

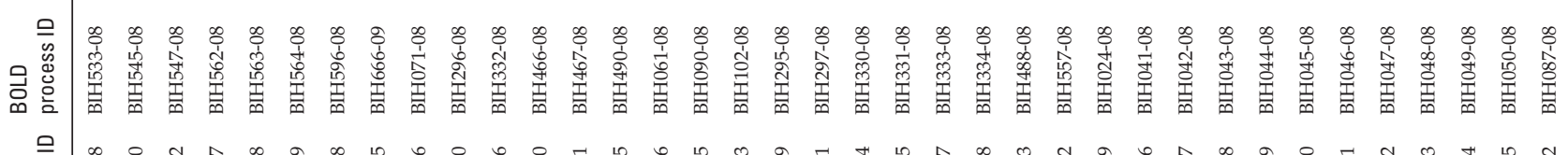

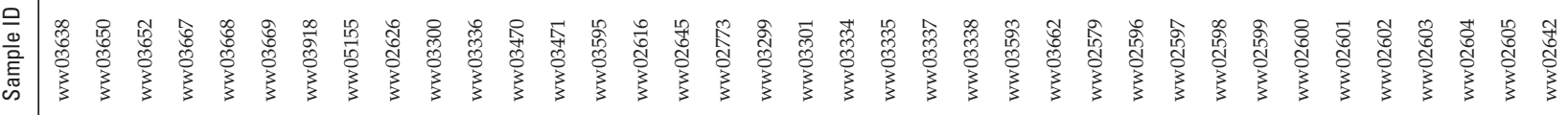




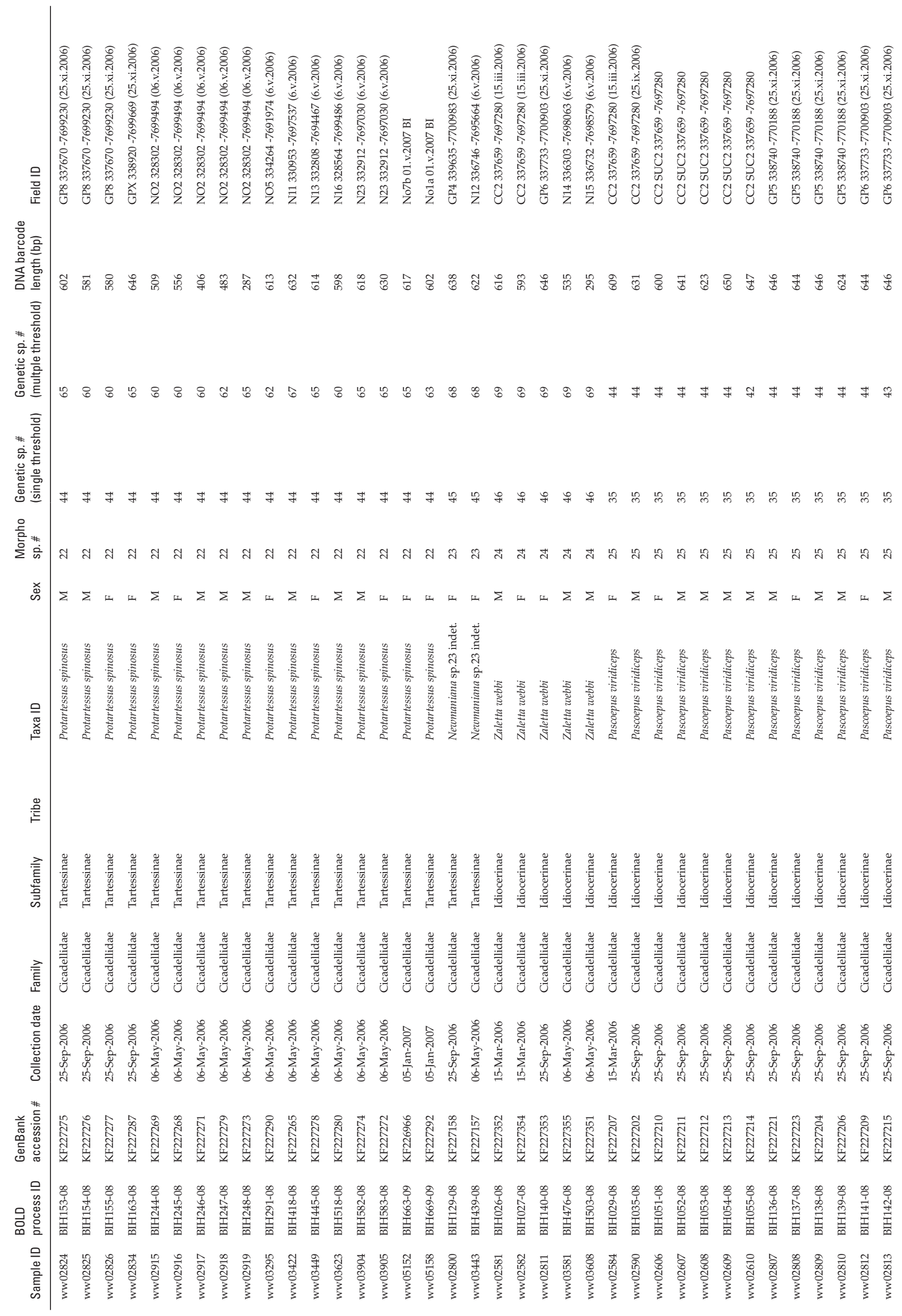


言

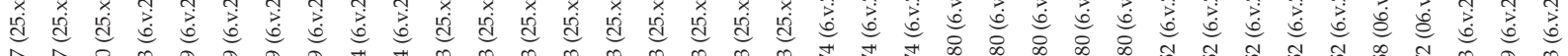

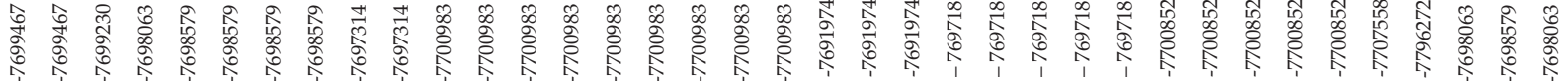

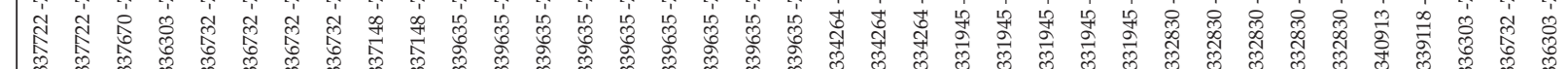

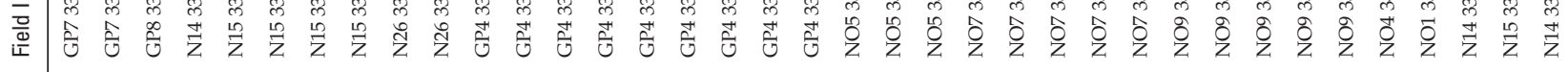

要要

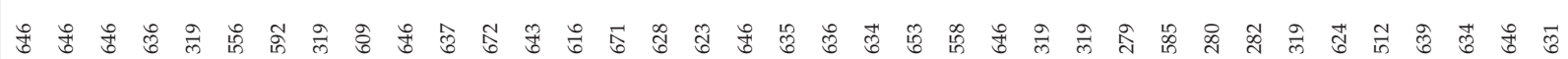

咅

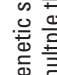

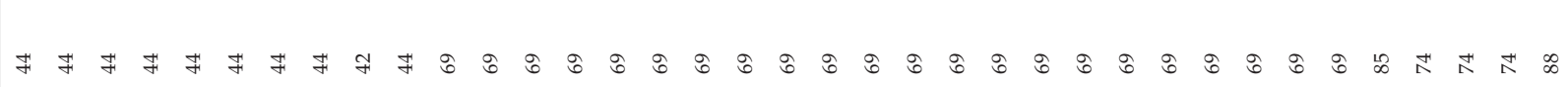

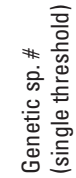

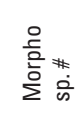

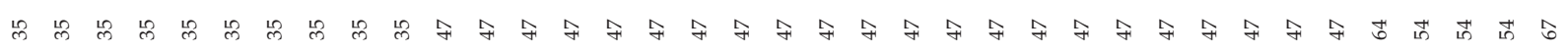

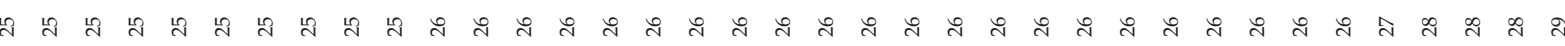

๙

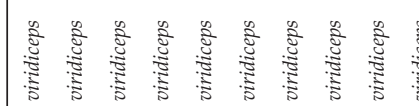

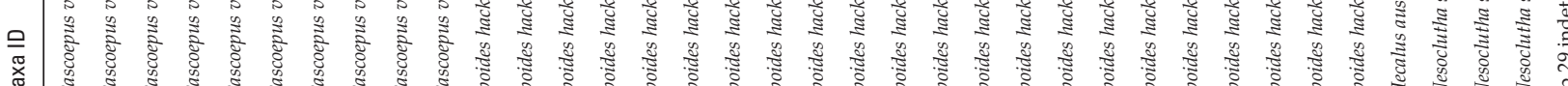

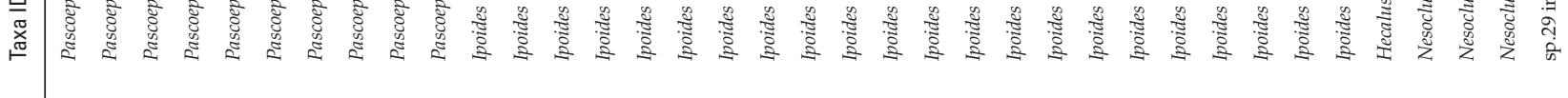

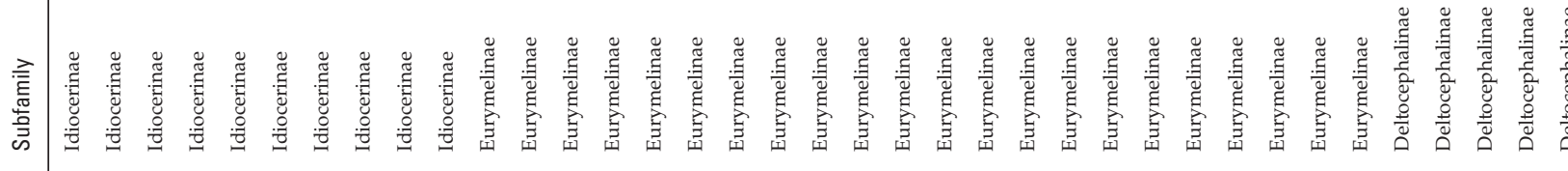

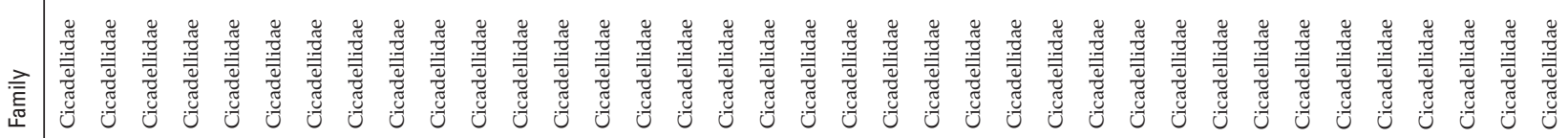

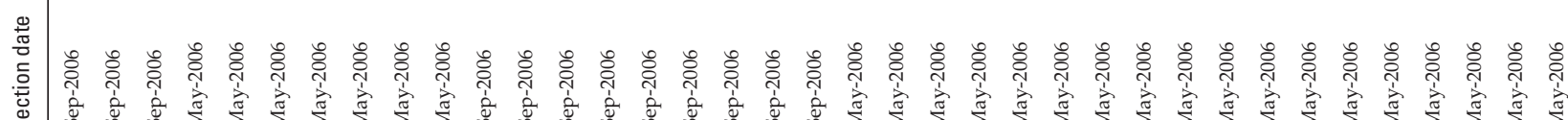

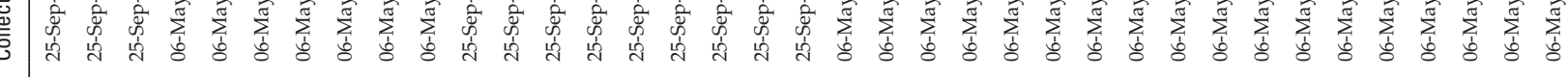

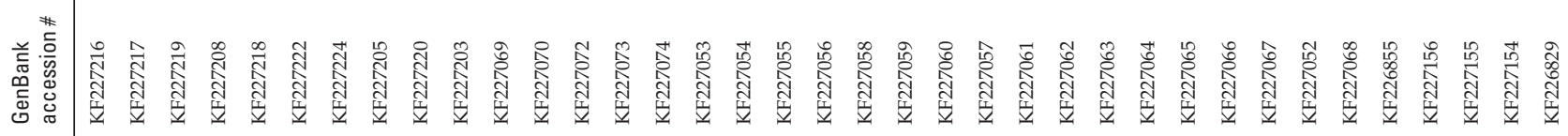

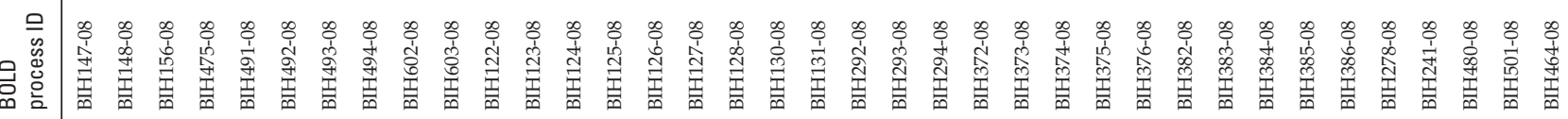

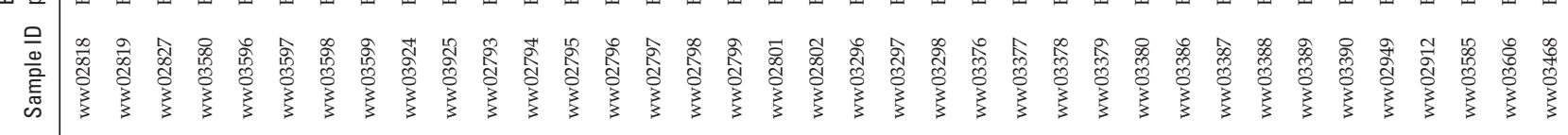




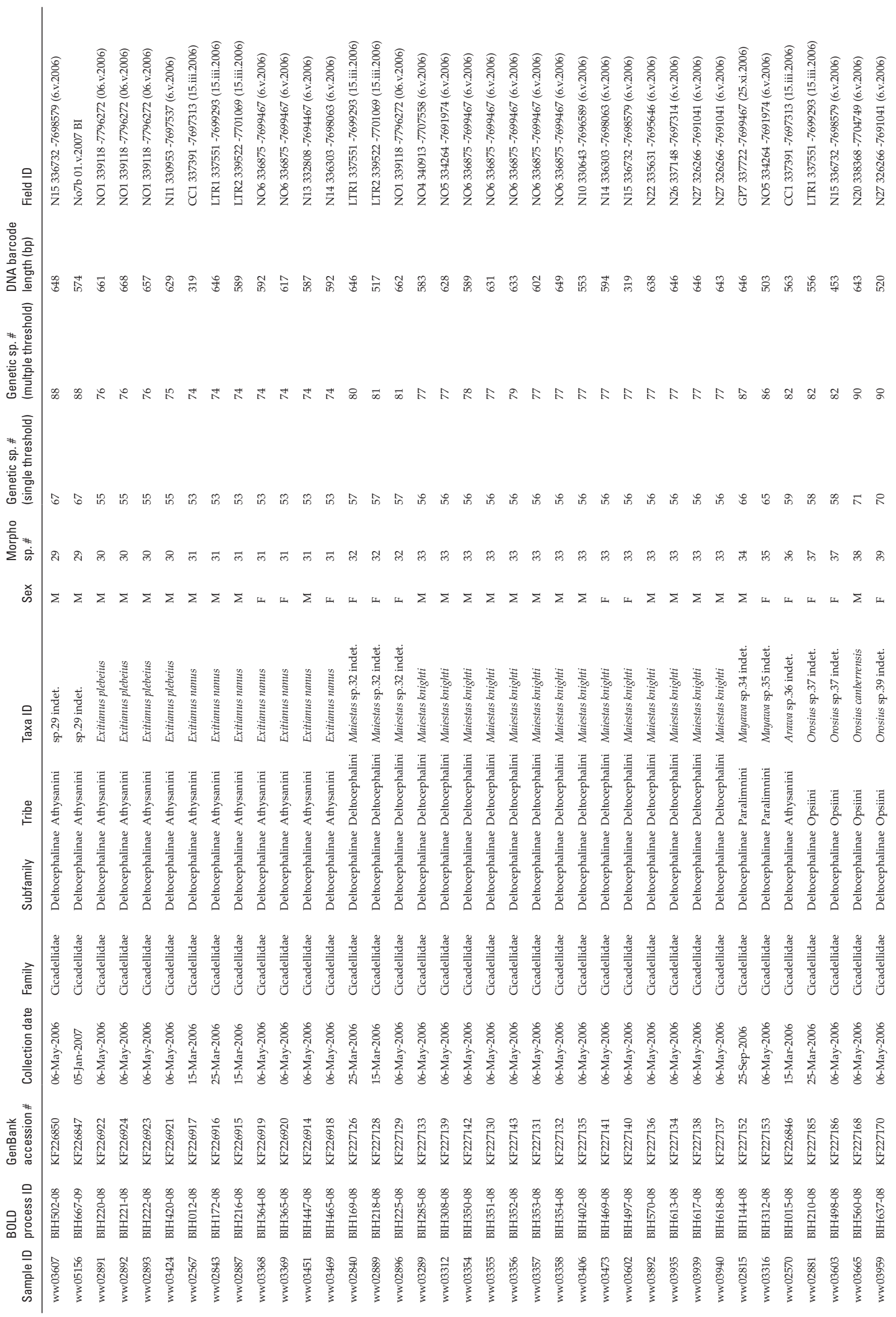




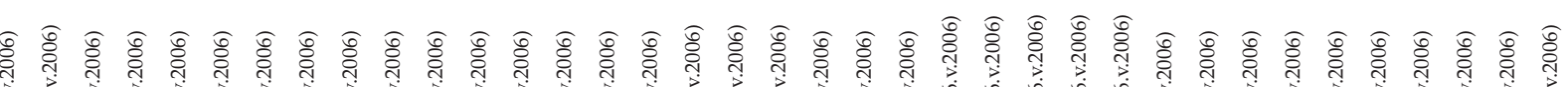

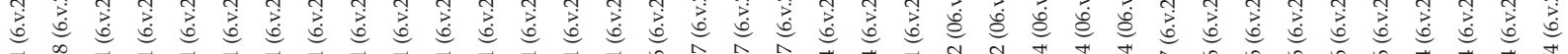

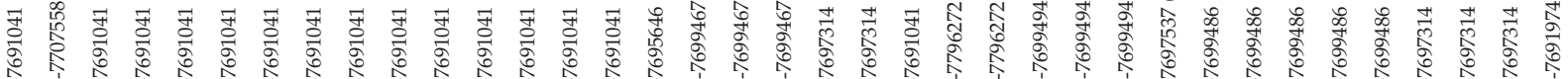

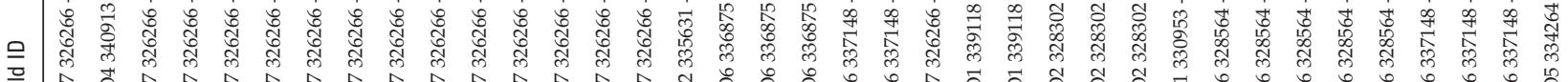

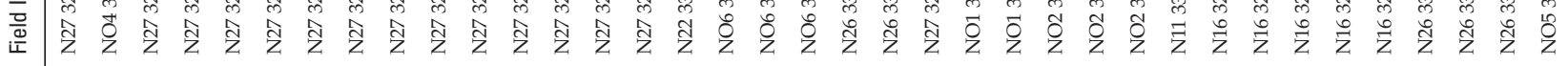

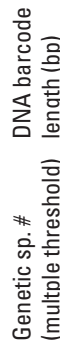

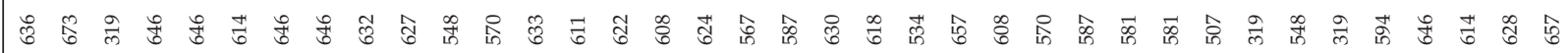

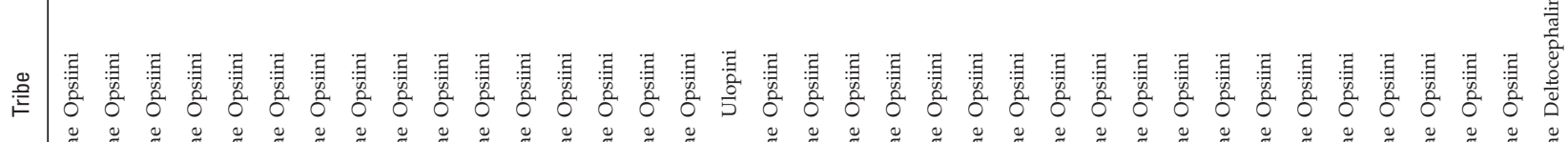

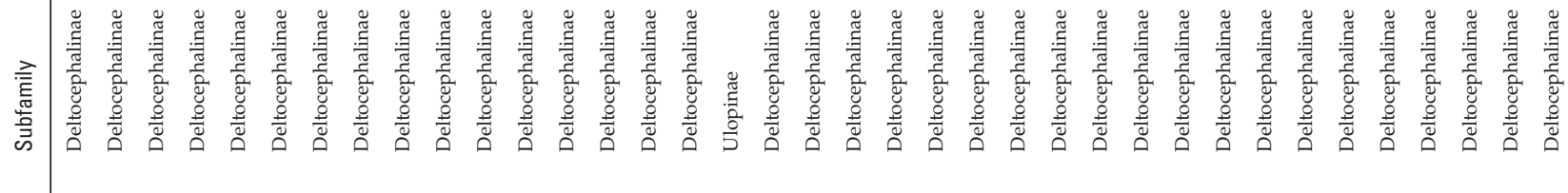

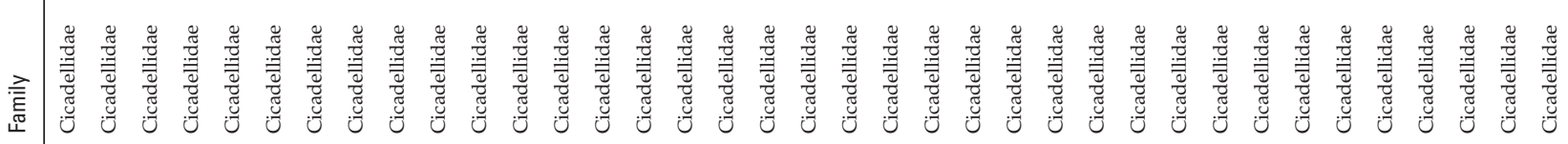
密

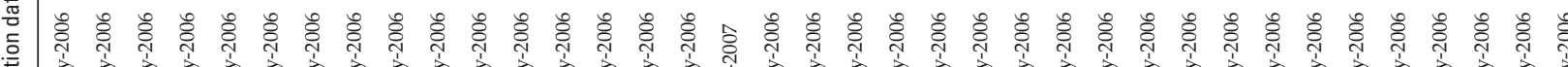

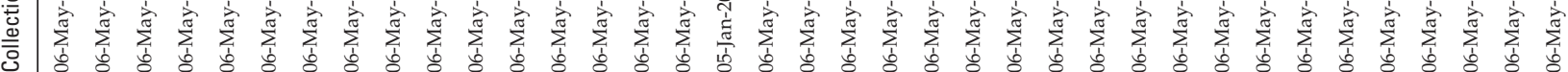

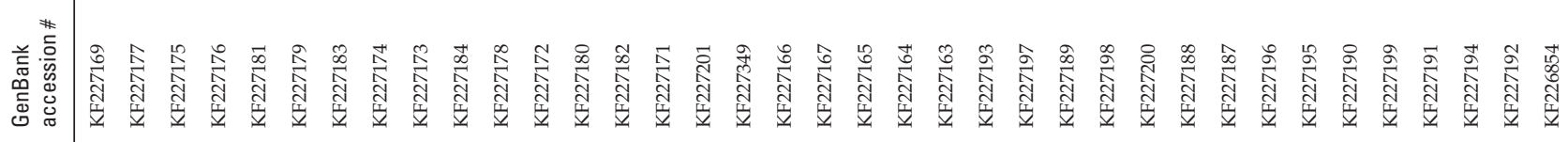

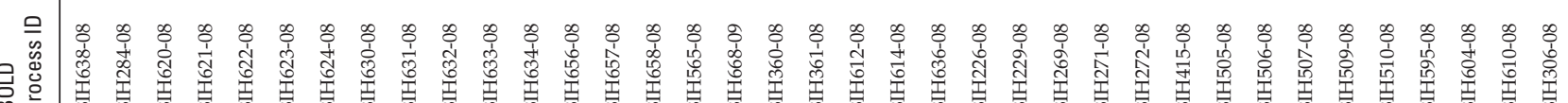

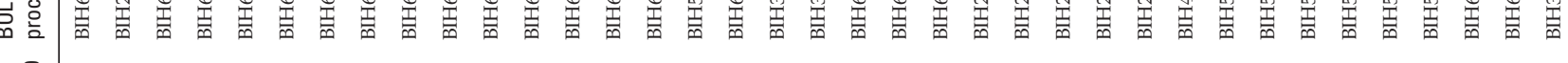

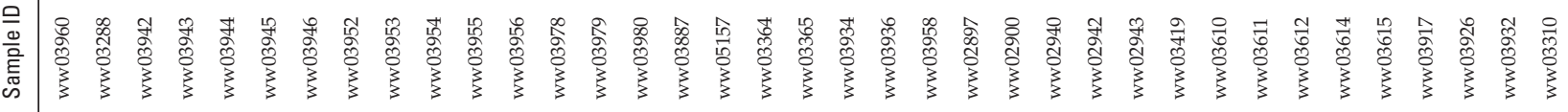




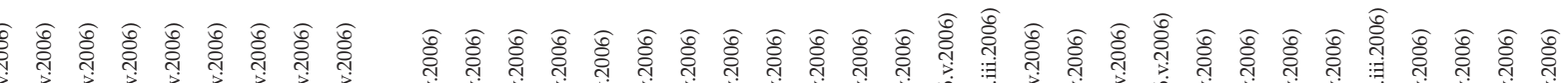

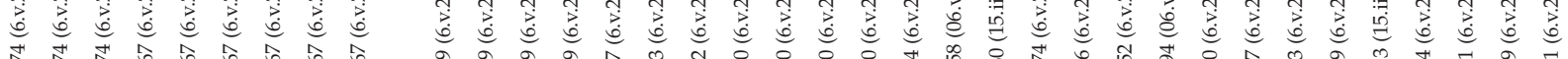

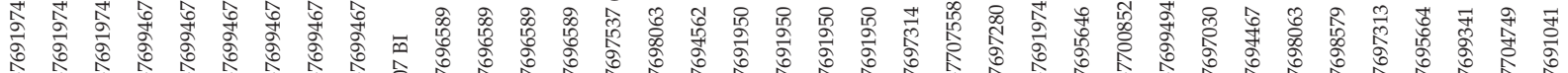

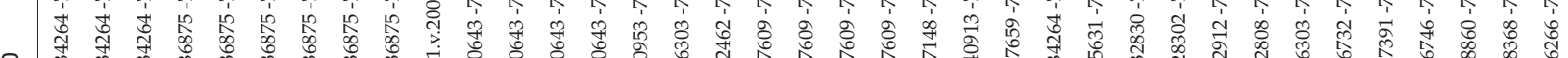

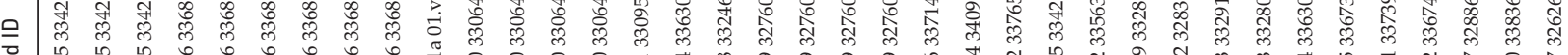

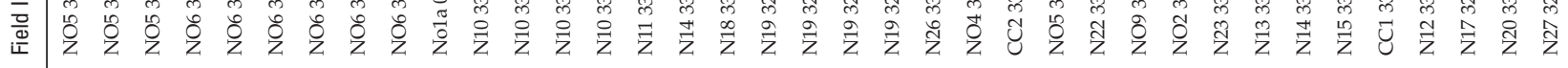

尊高

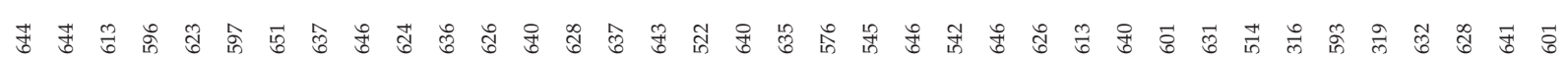

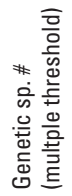

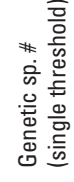

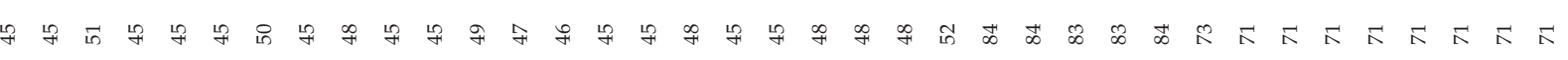

訔

×

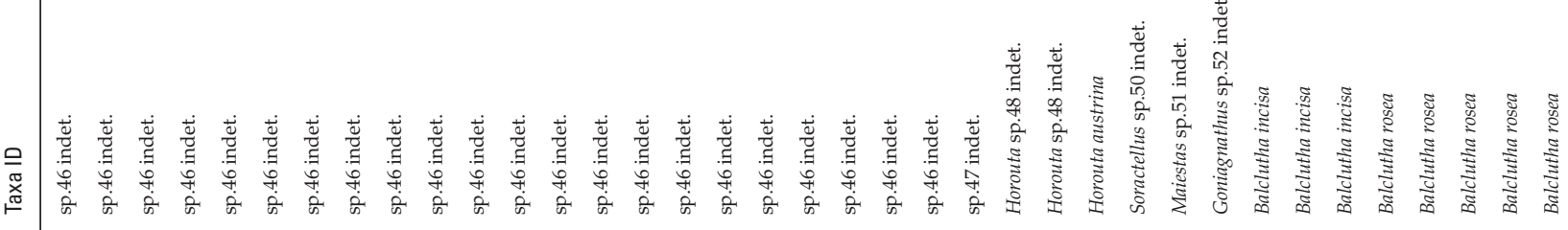

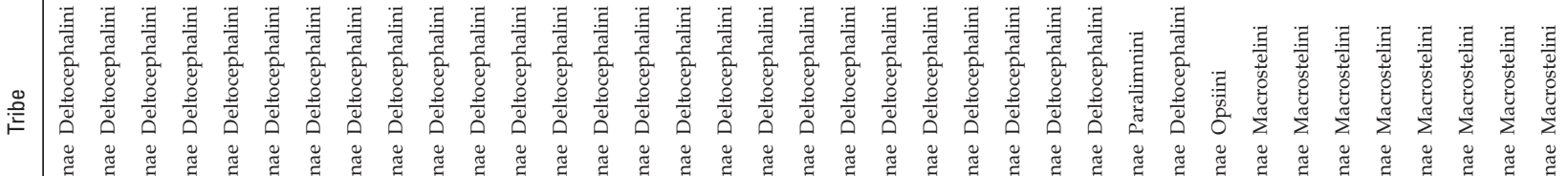

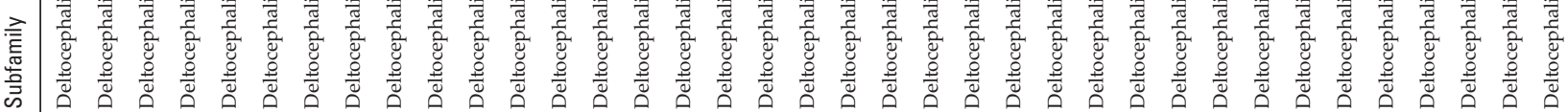

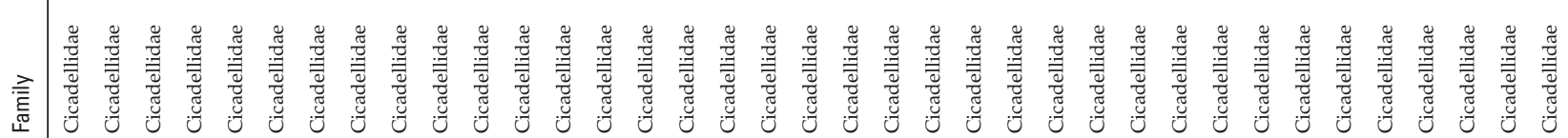

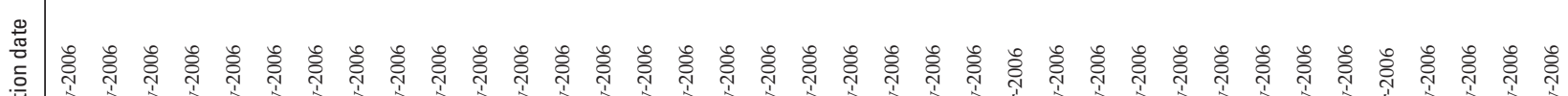

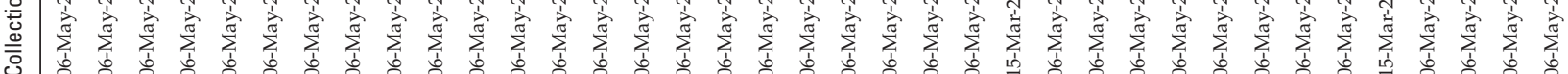

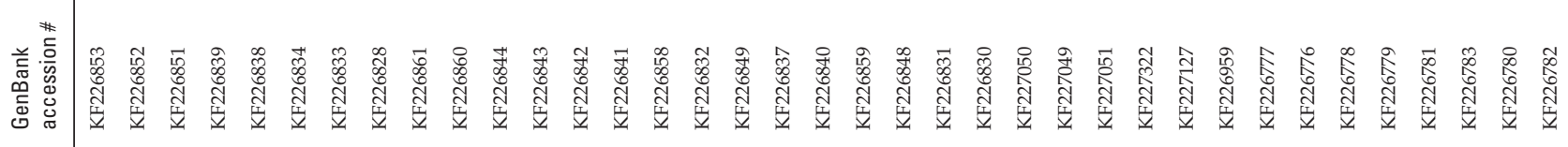

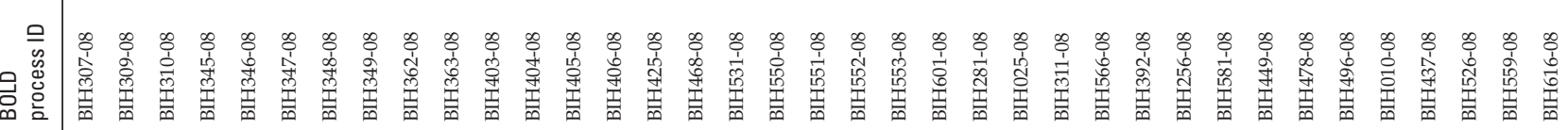

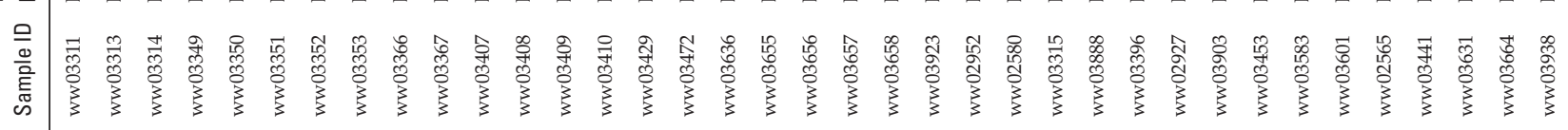




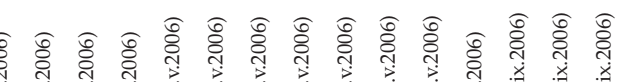

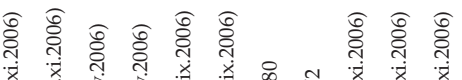

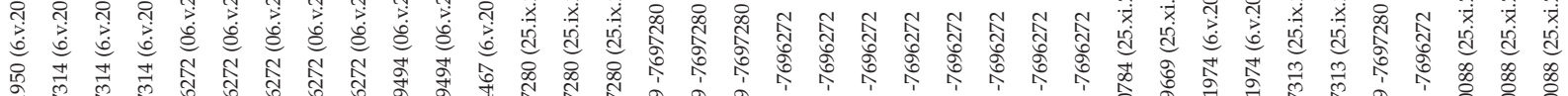

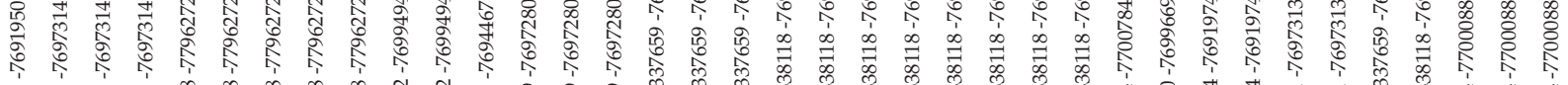

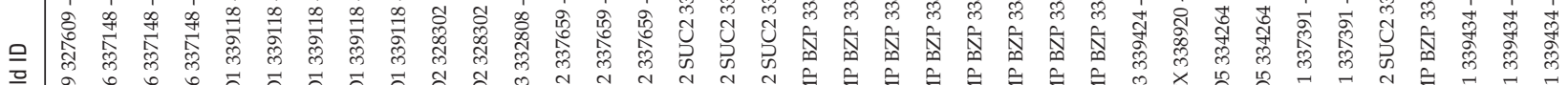

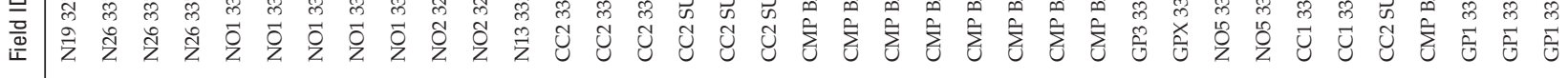

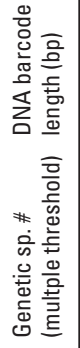

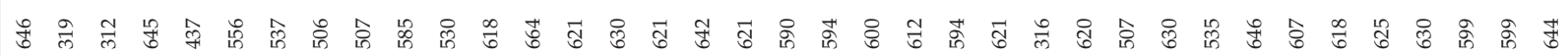

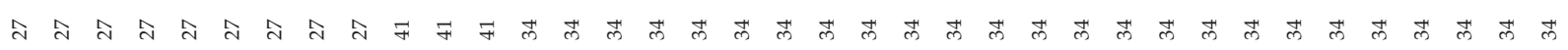

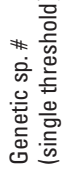

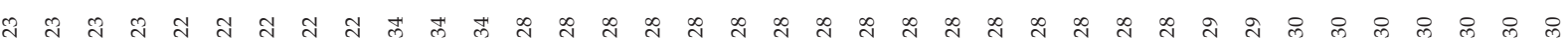

을

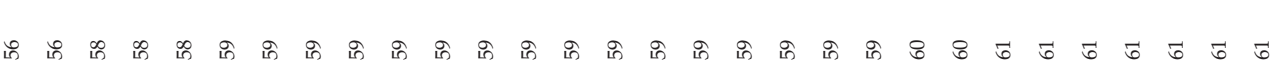
$\Sigma \Sigma \Sigma$

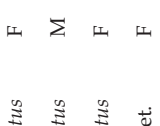

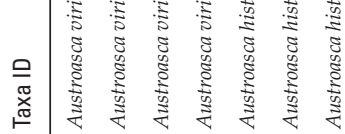

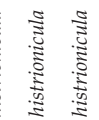

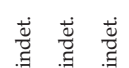

䒜

苋

选

䗢

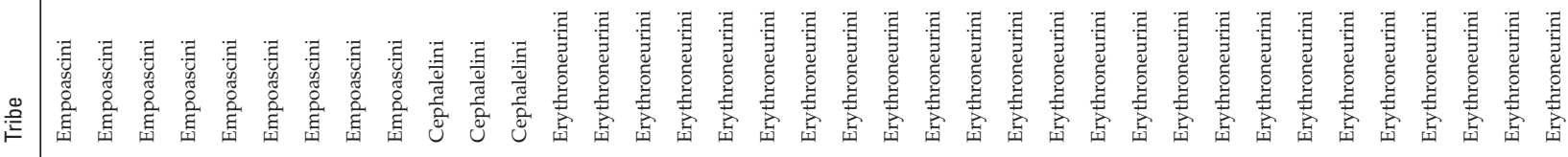

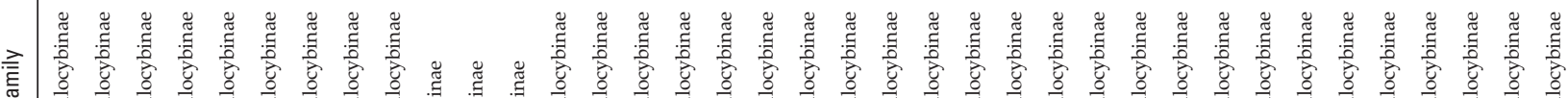

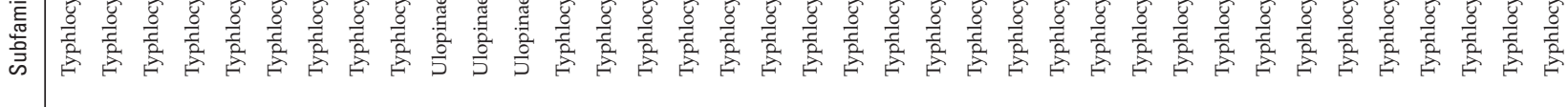

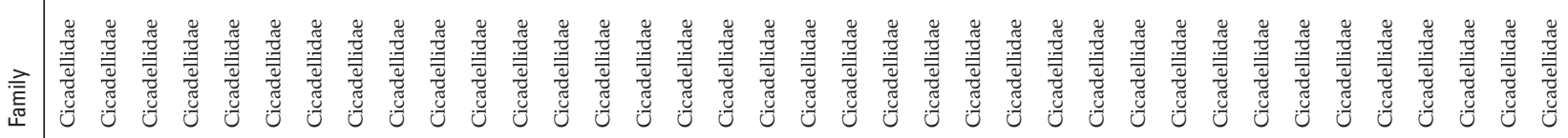

密

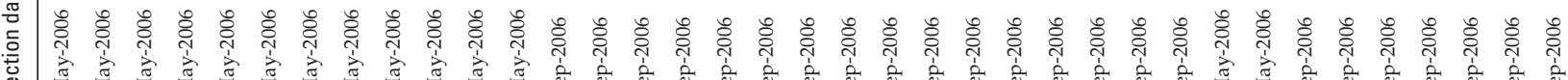

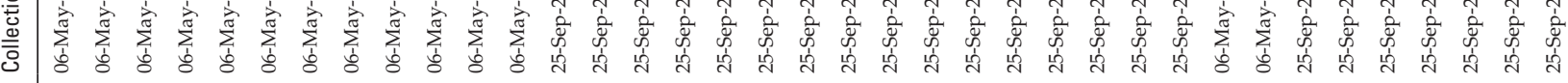

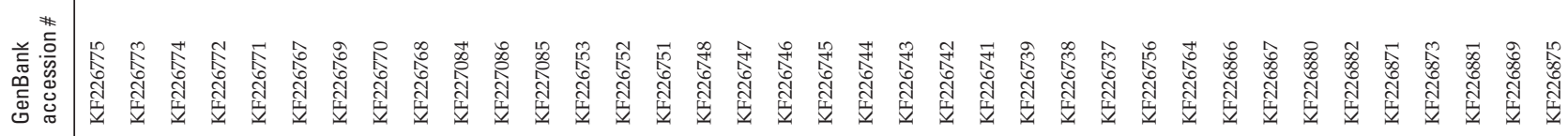

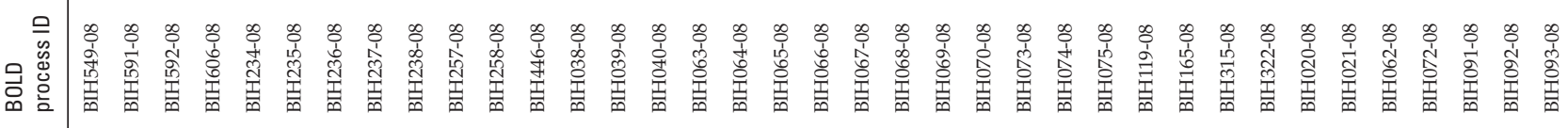

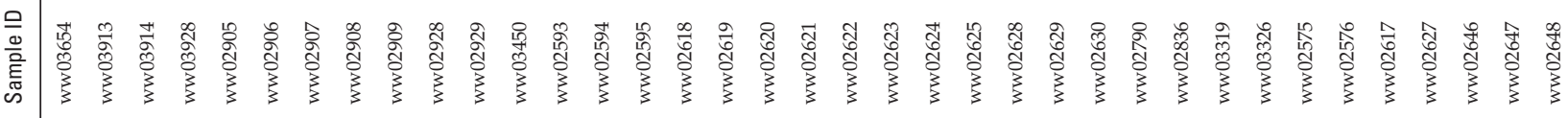




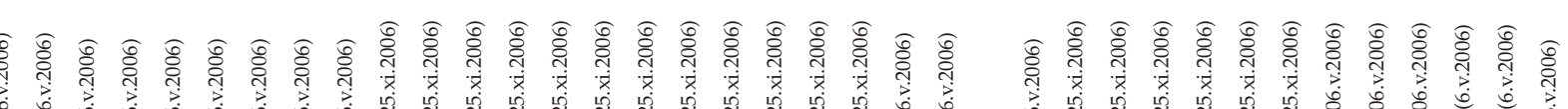

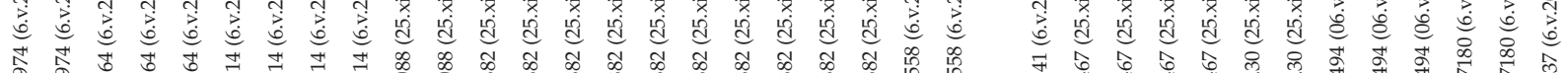

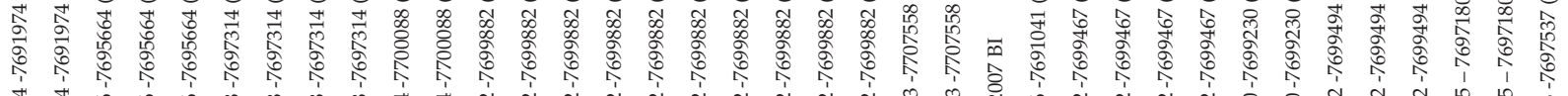

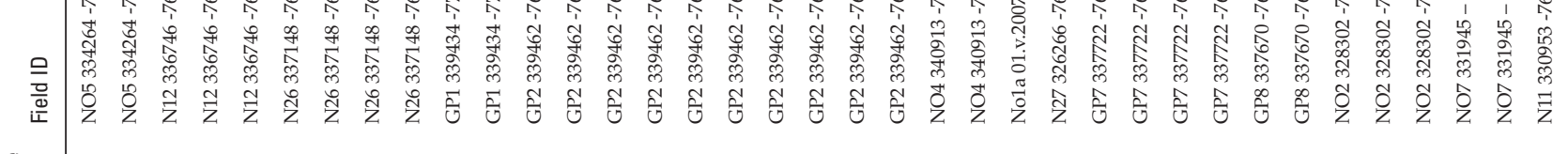

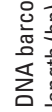
ชิ

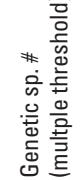

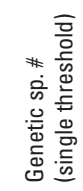

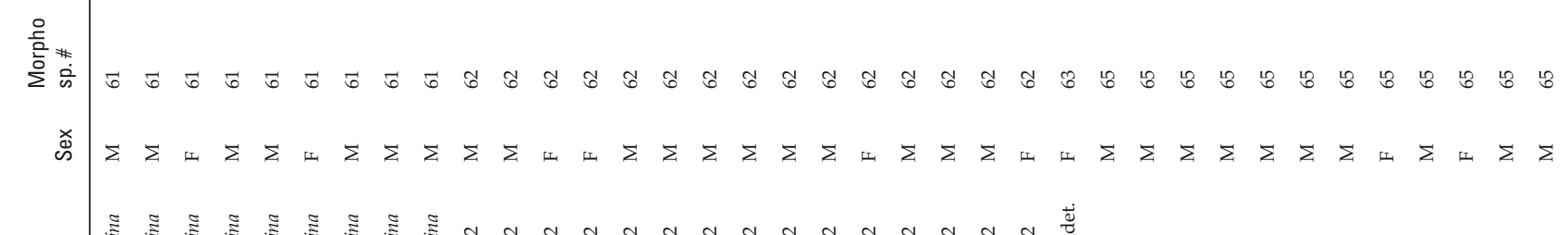

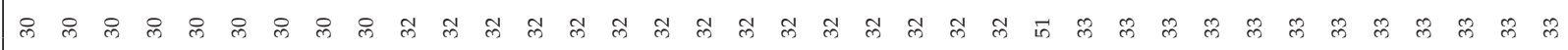

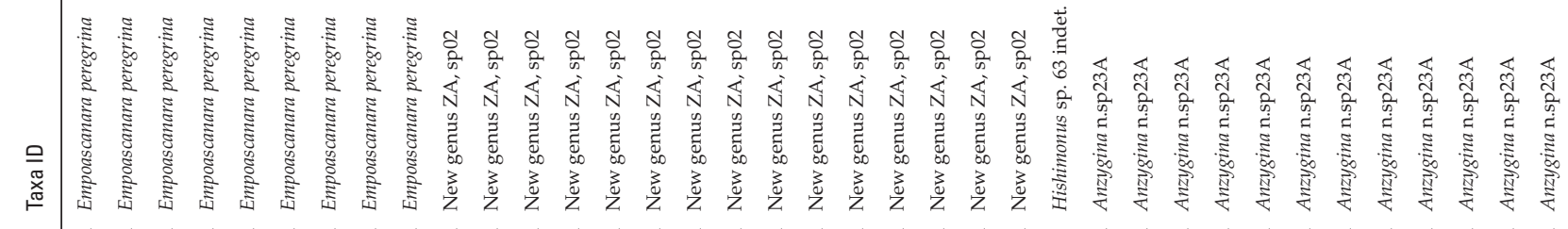

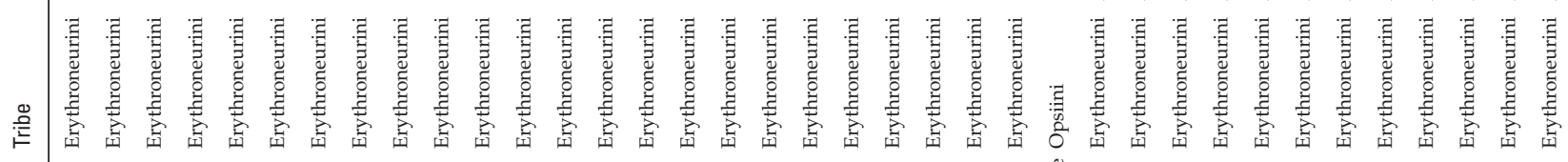

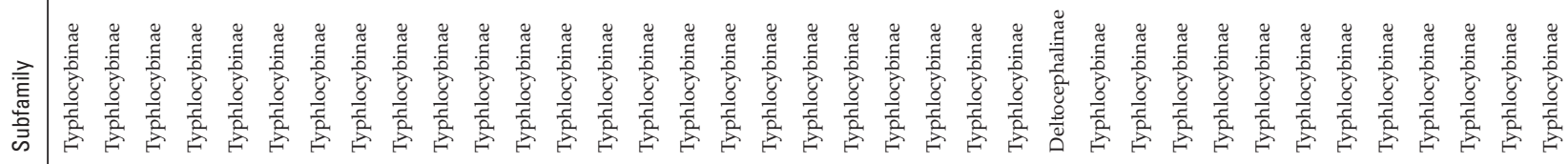

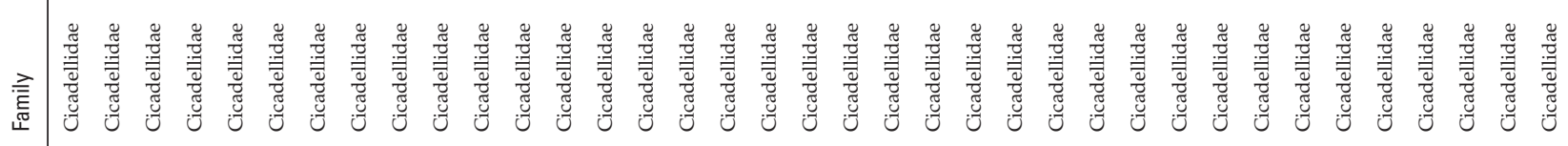
站

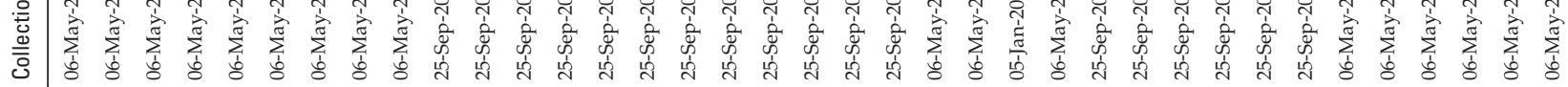

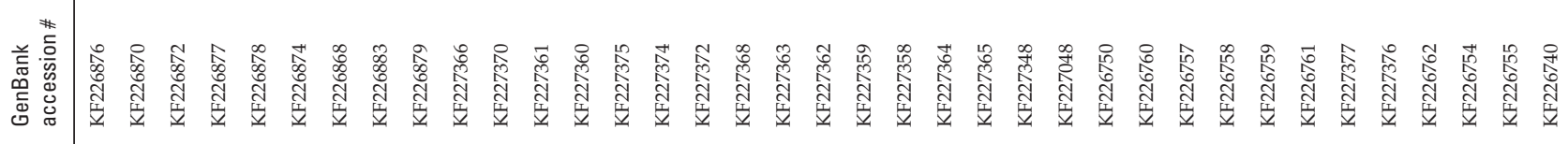

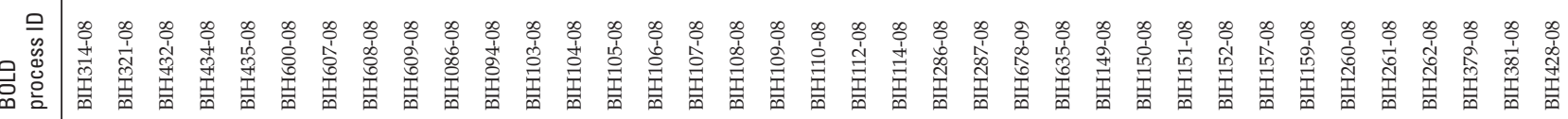

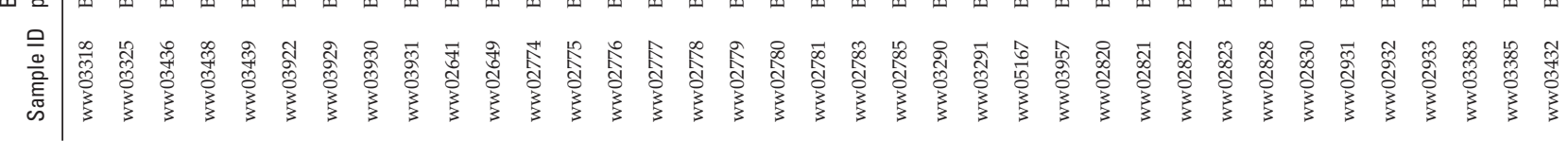


ठ

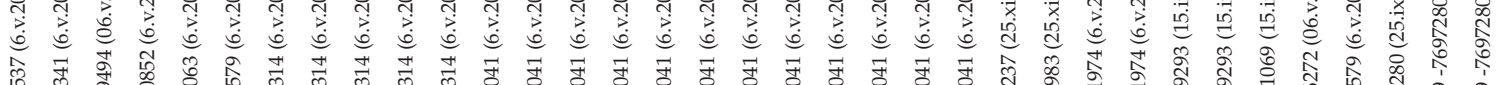

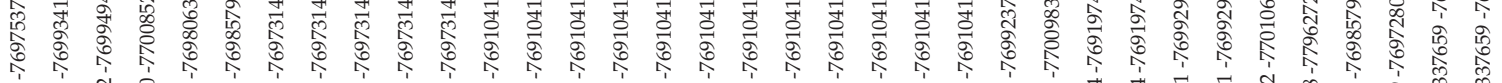

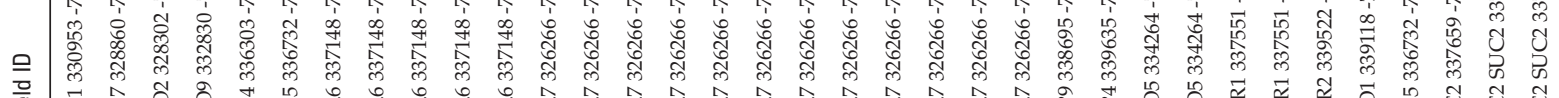

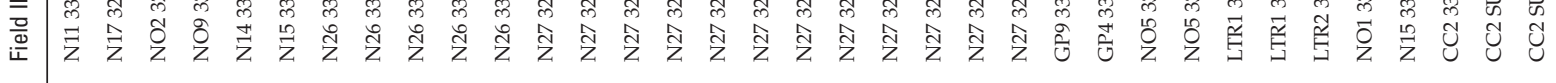

要要

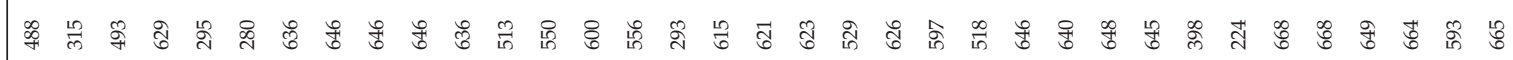

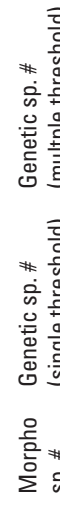

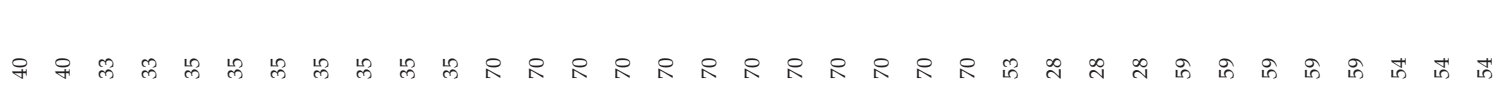

* $\frac{\bar{c}}{\frac{0}{0}}$

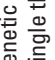

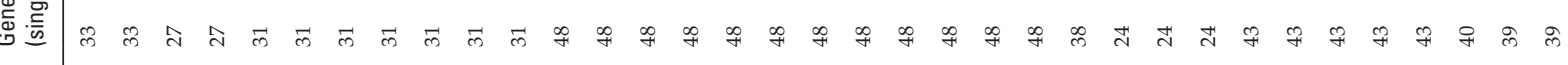

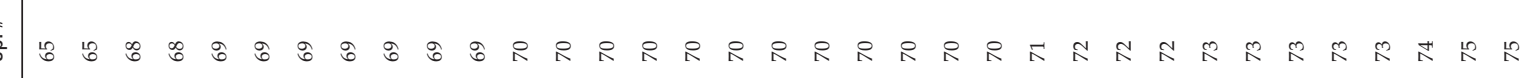
œ

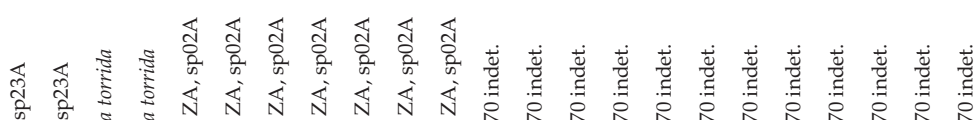

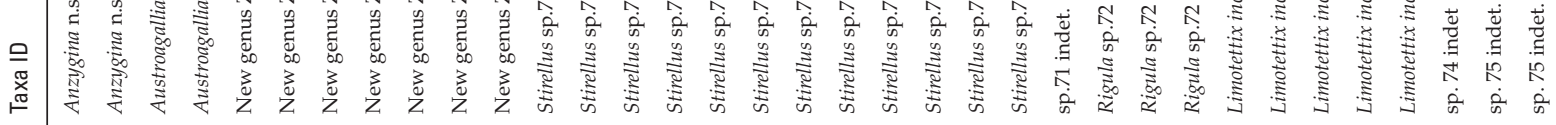

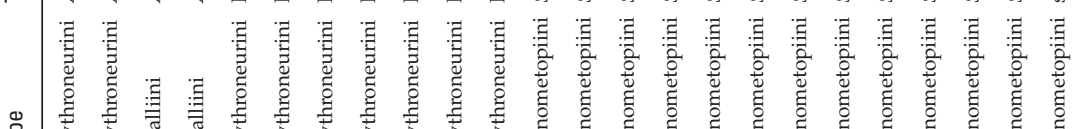

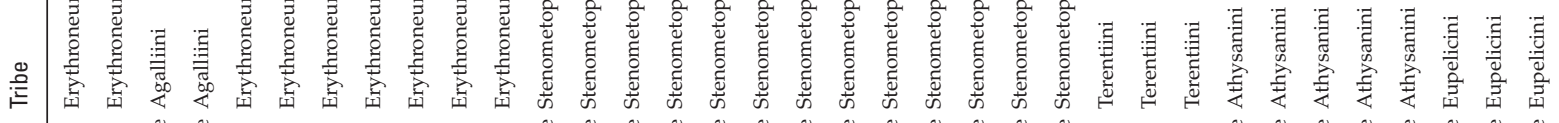

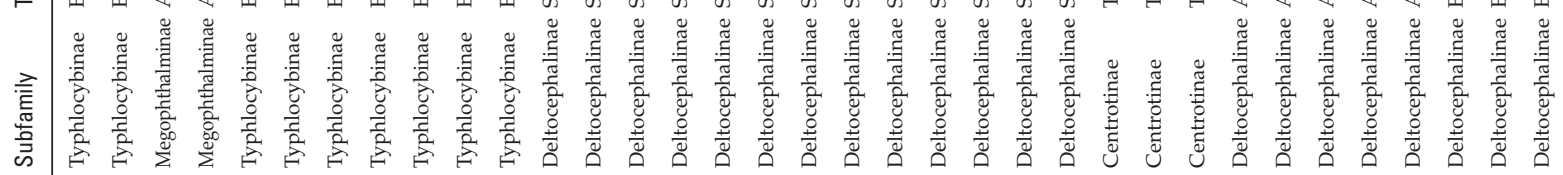

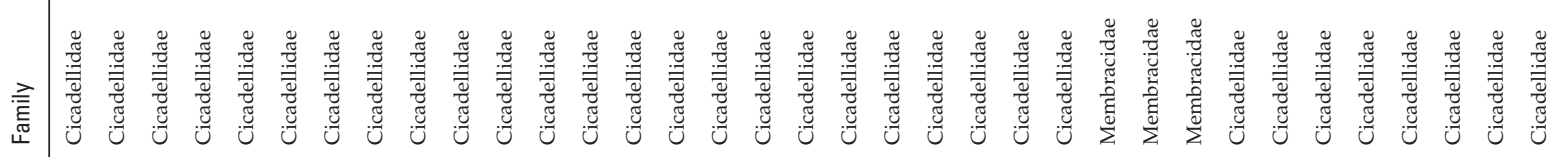

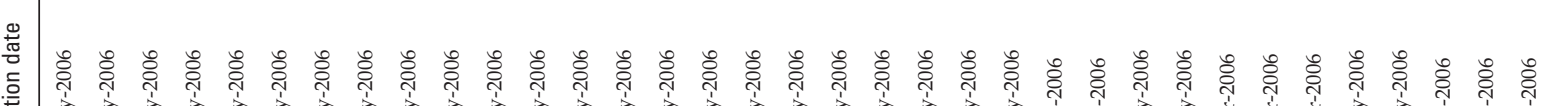

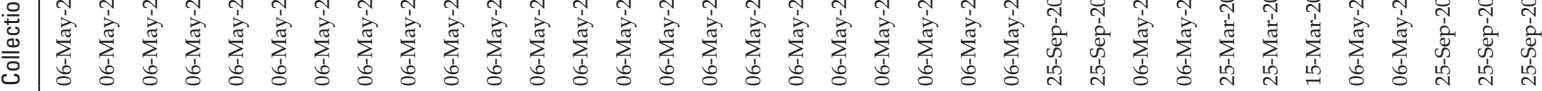

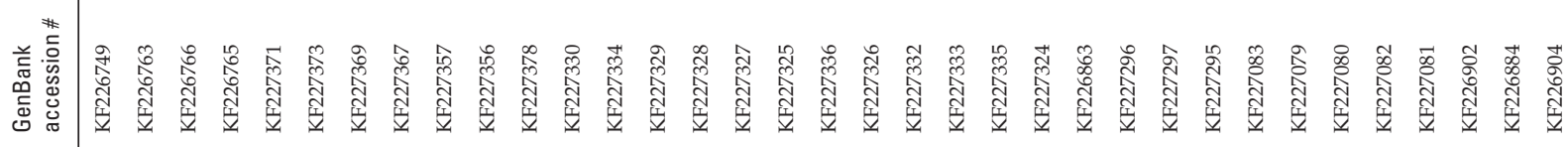

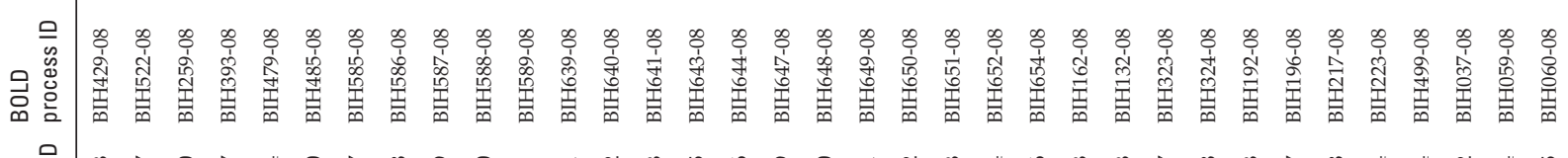

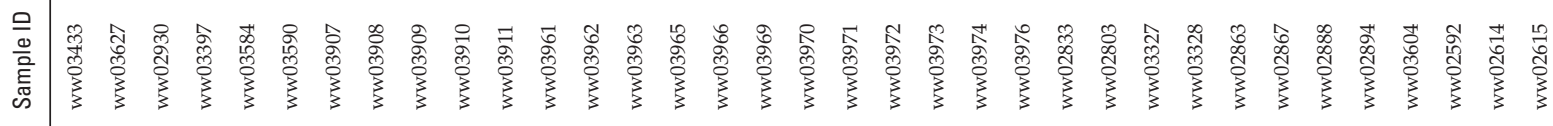




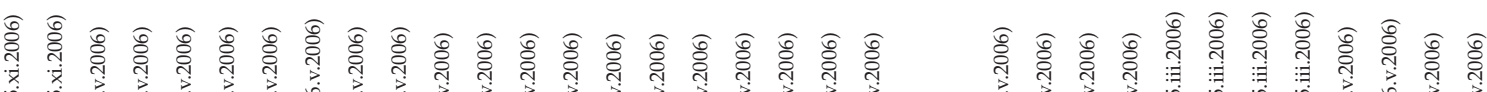

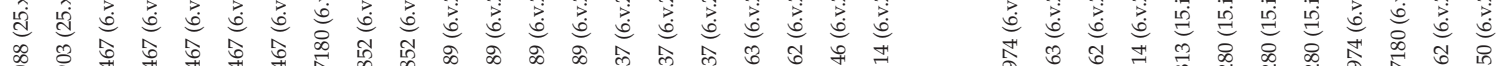

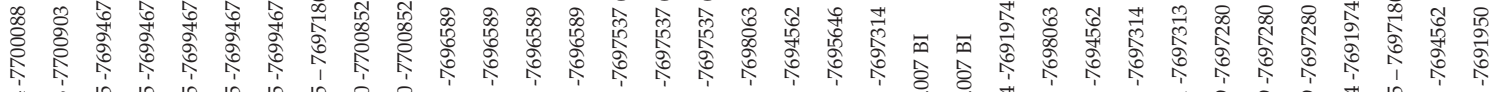

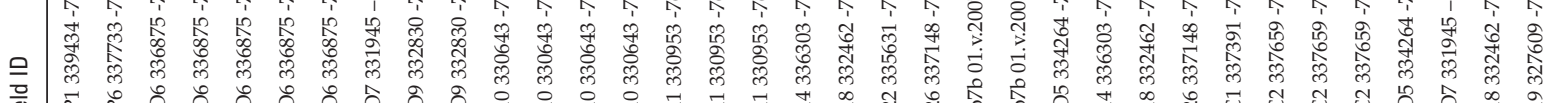

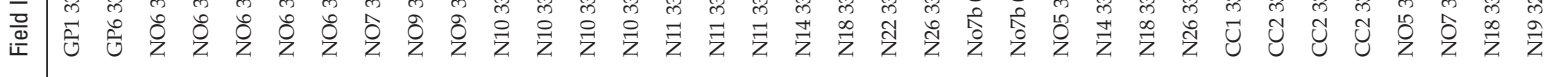

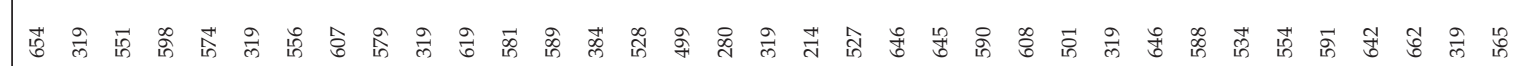

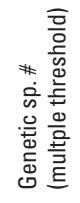

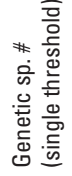

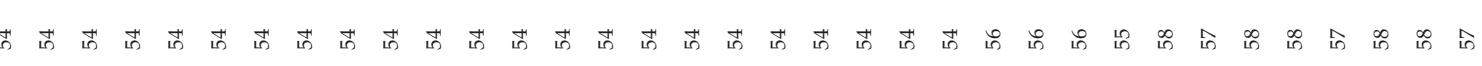

을 के के के

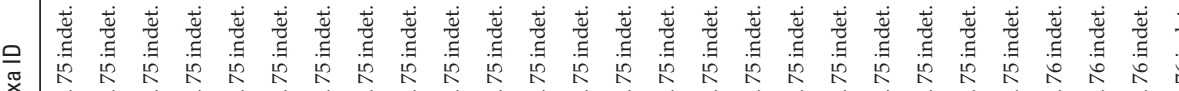

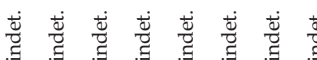

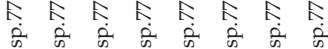

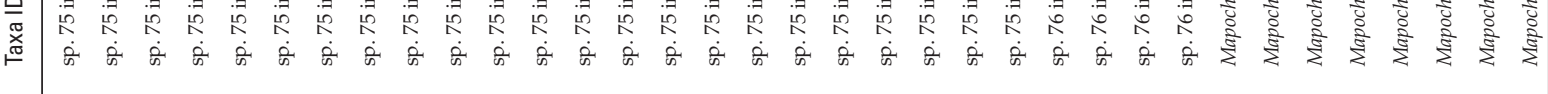

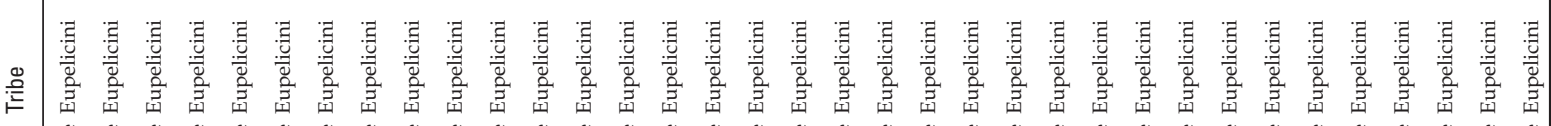

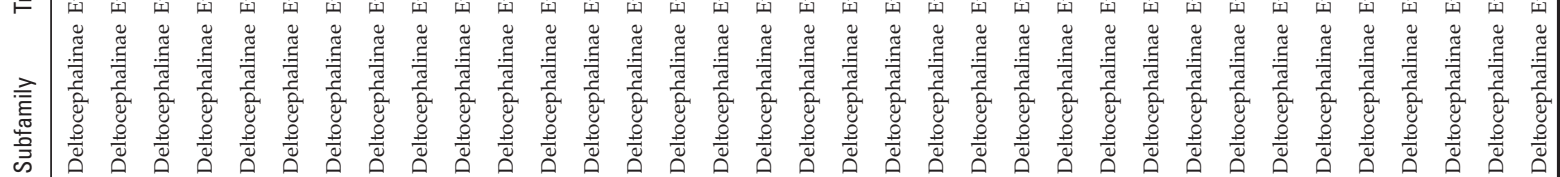

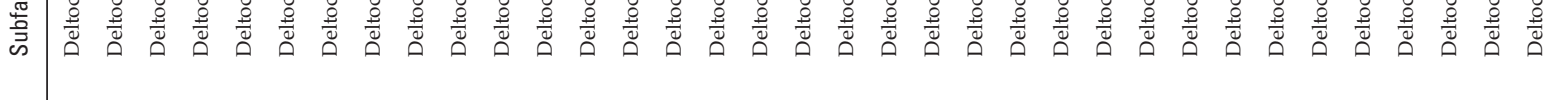

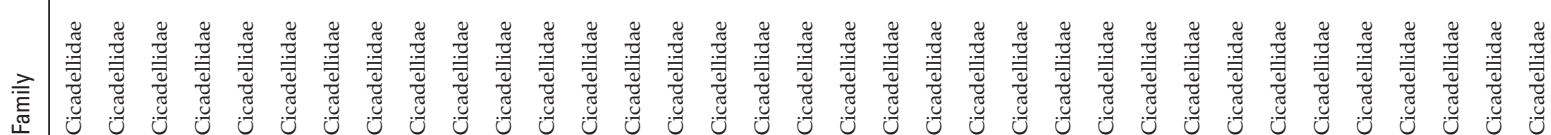
兽

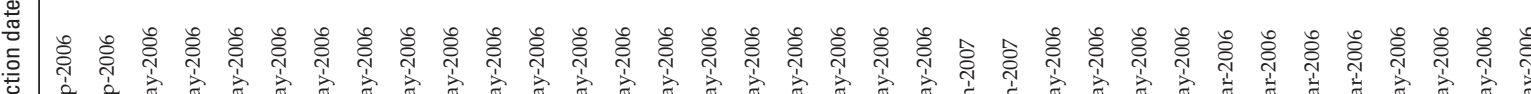

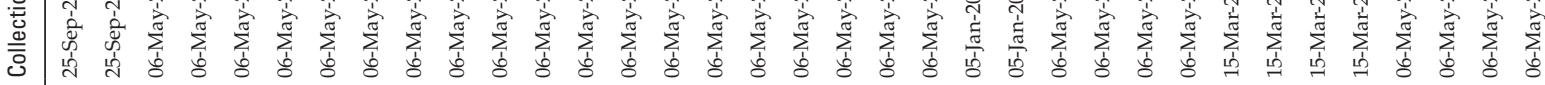

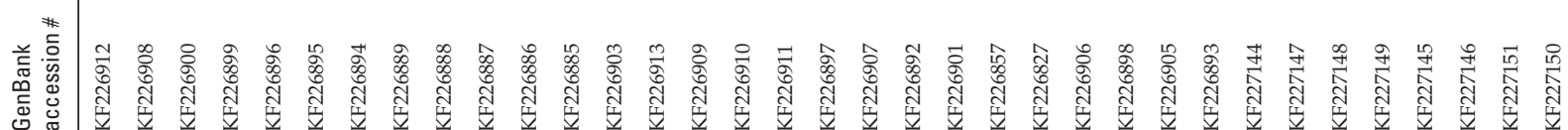

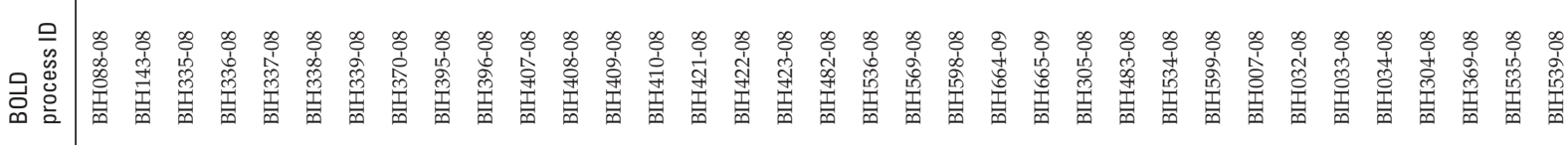

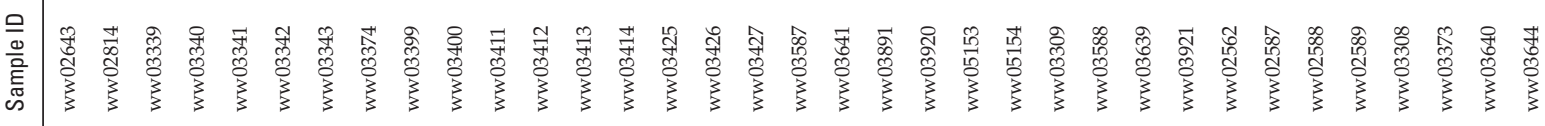


$\frac{\pi}{5} \stackrel{0}{\frac{3}{0}} \frac{0}{0}$

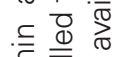

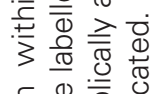

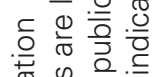

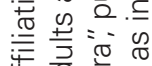

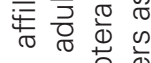

을 을

巴)

ब售

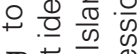

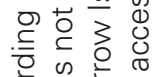

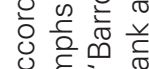

ह

过

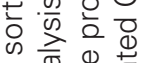

क $\frac{1}{\pi} \frac{0}{0}$

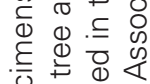

की. 일 엄

i) 옹 다

ट

हो 당

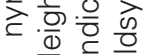

중 $\frac{1}{2}=\frac{0}{0}$

단.

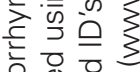

헝혀응

응 눙ㅇㅇ

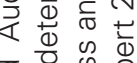

응 is 0

$\frac{\pi}{0} \cdot \frac{0}{0}$ 인

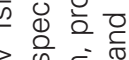

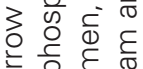

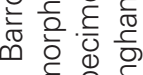

व है के ज

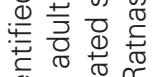

응 우 흉뜬

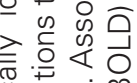

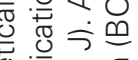

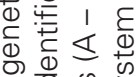

4.00

年

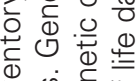

㐫

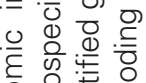

है की

ํํㅇㅎㅇ음

离

$\underset{\substack{\bar{x} \\ \frac{\mathrm{a}}{\mathrm{a}}}}{\frac{\bar{x}}{4}}$

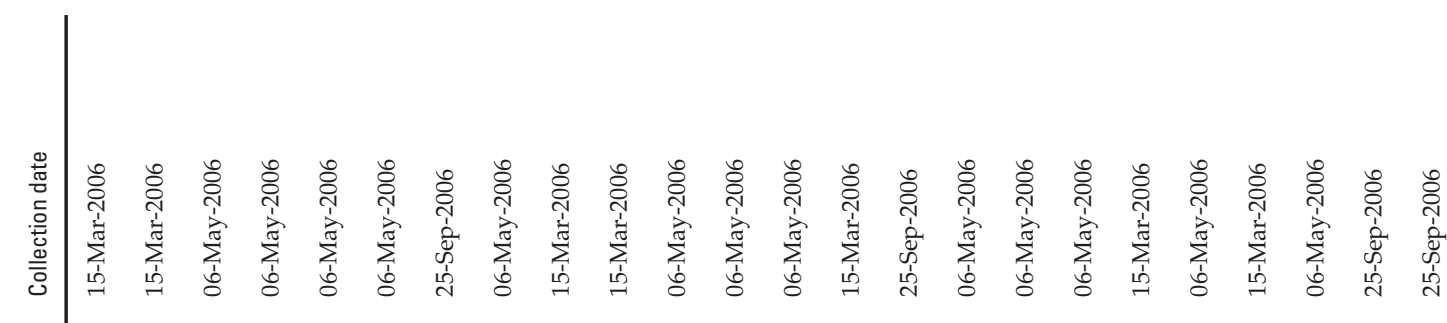

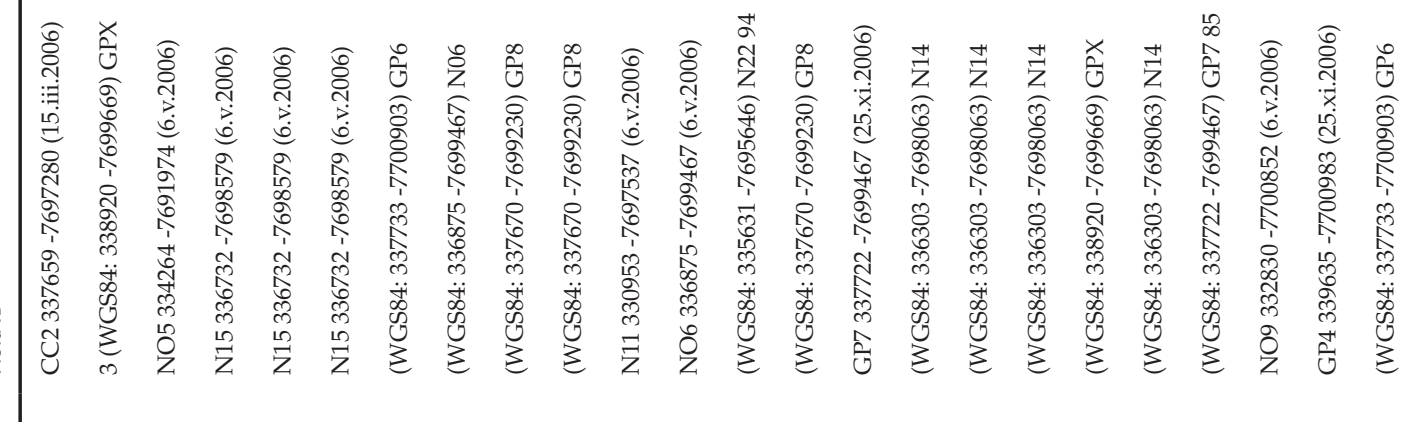

융 兽 त్ర

\section{蓠}

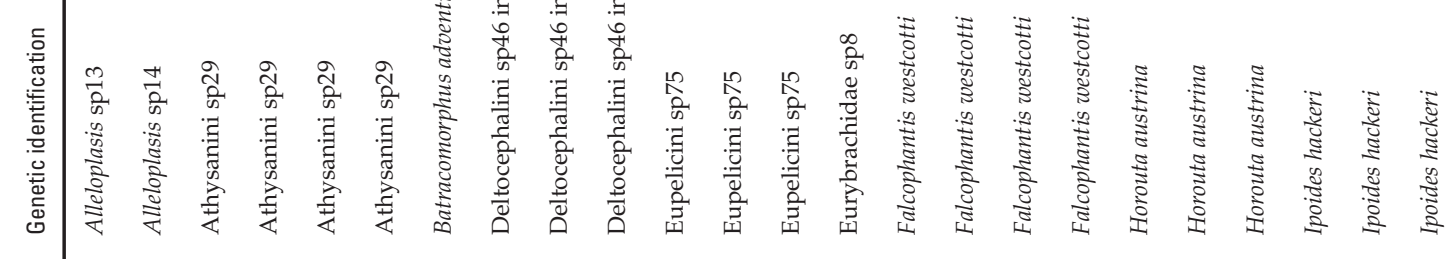

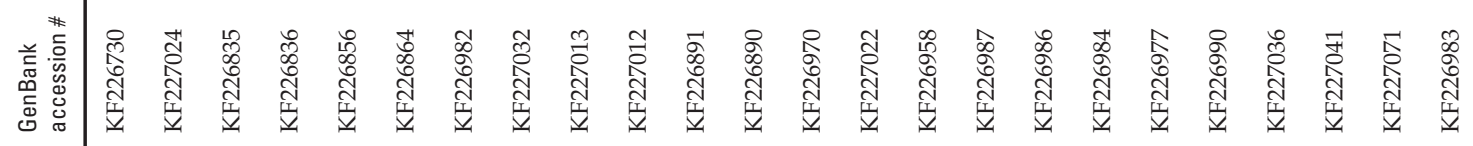

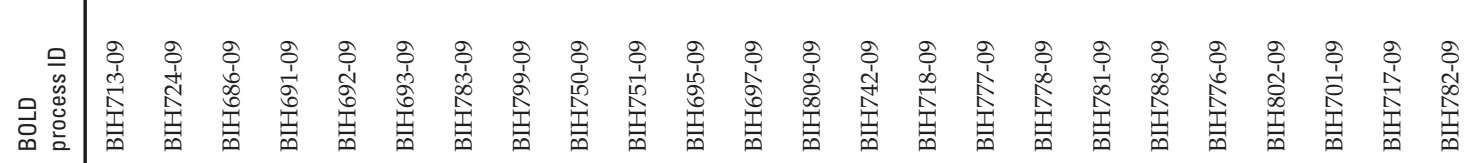

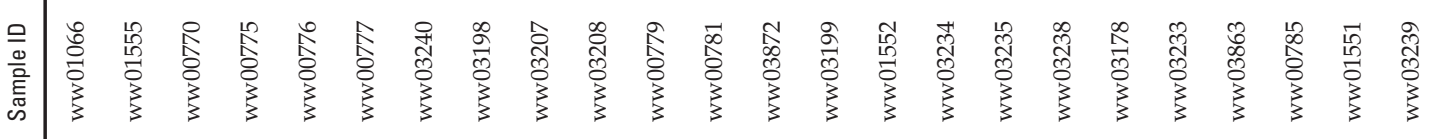




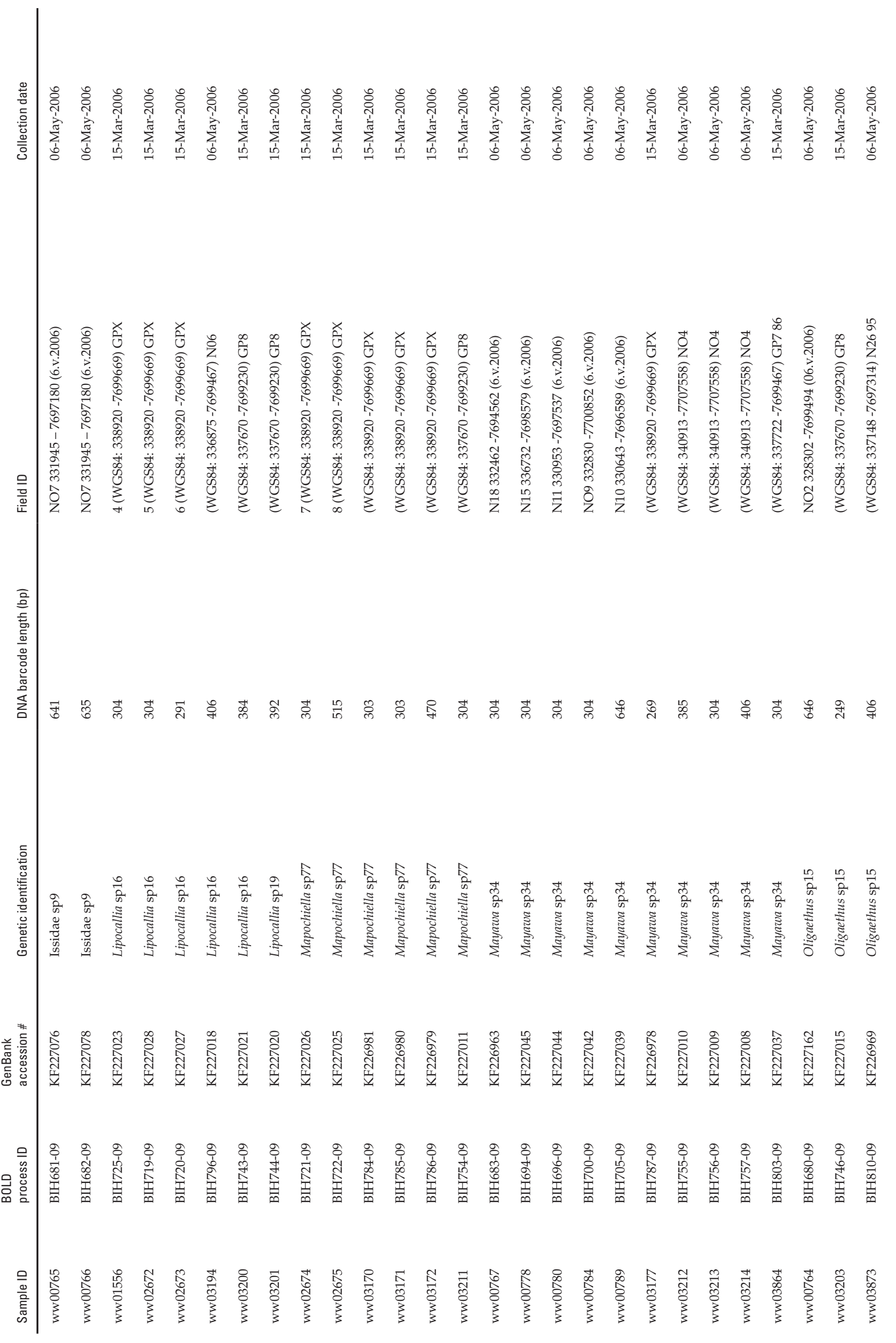




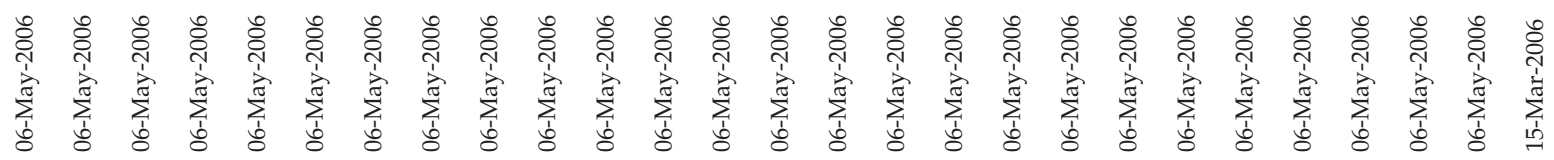

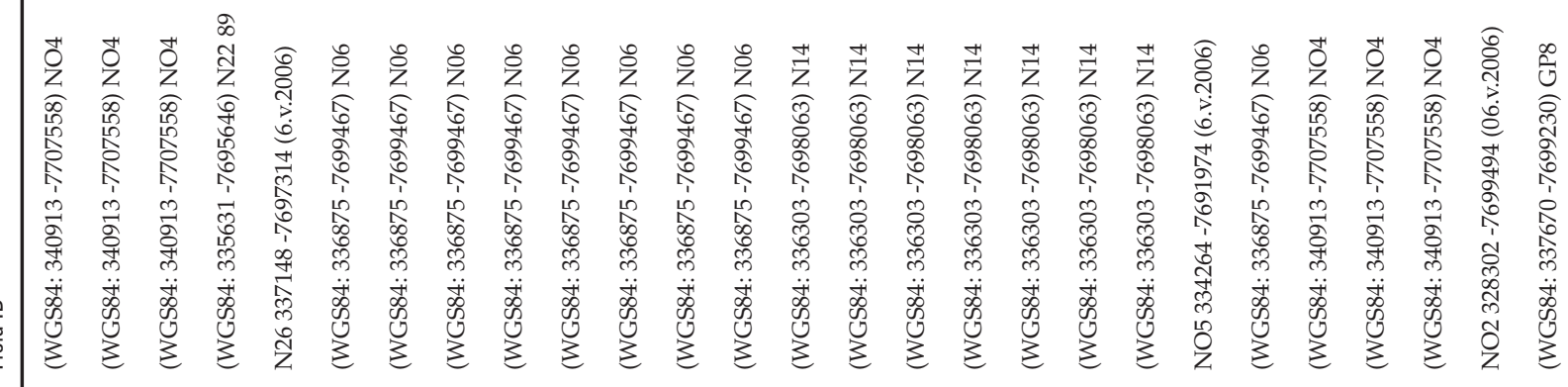

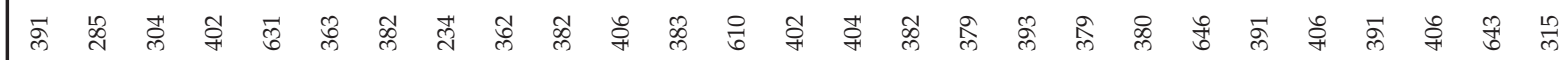

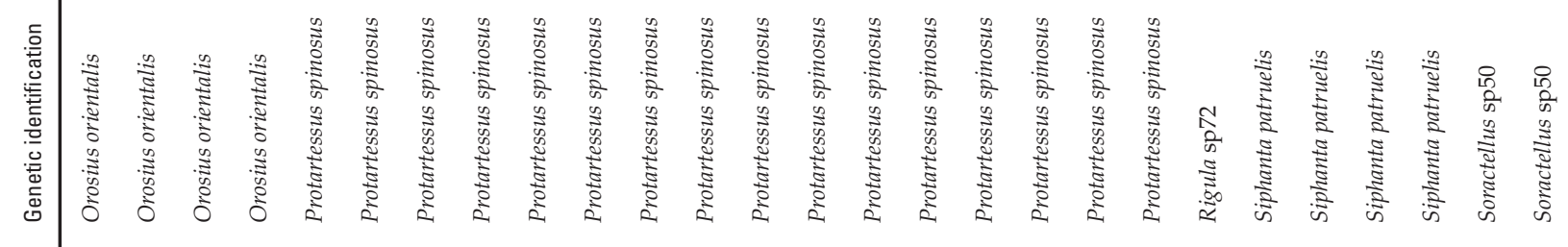

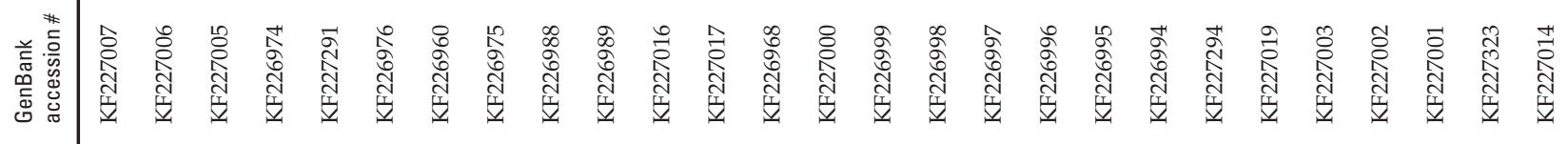

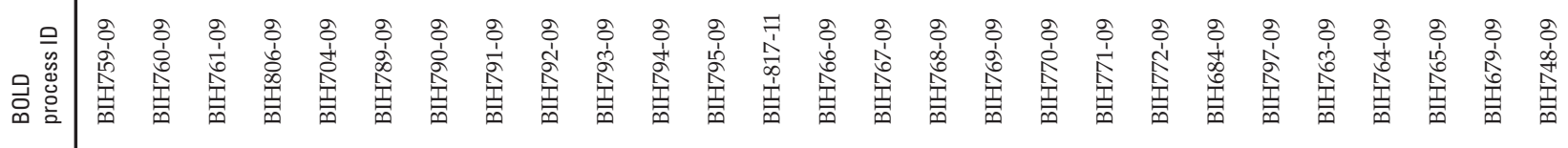

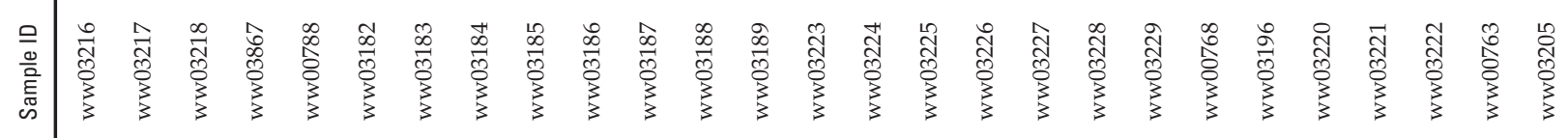




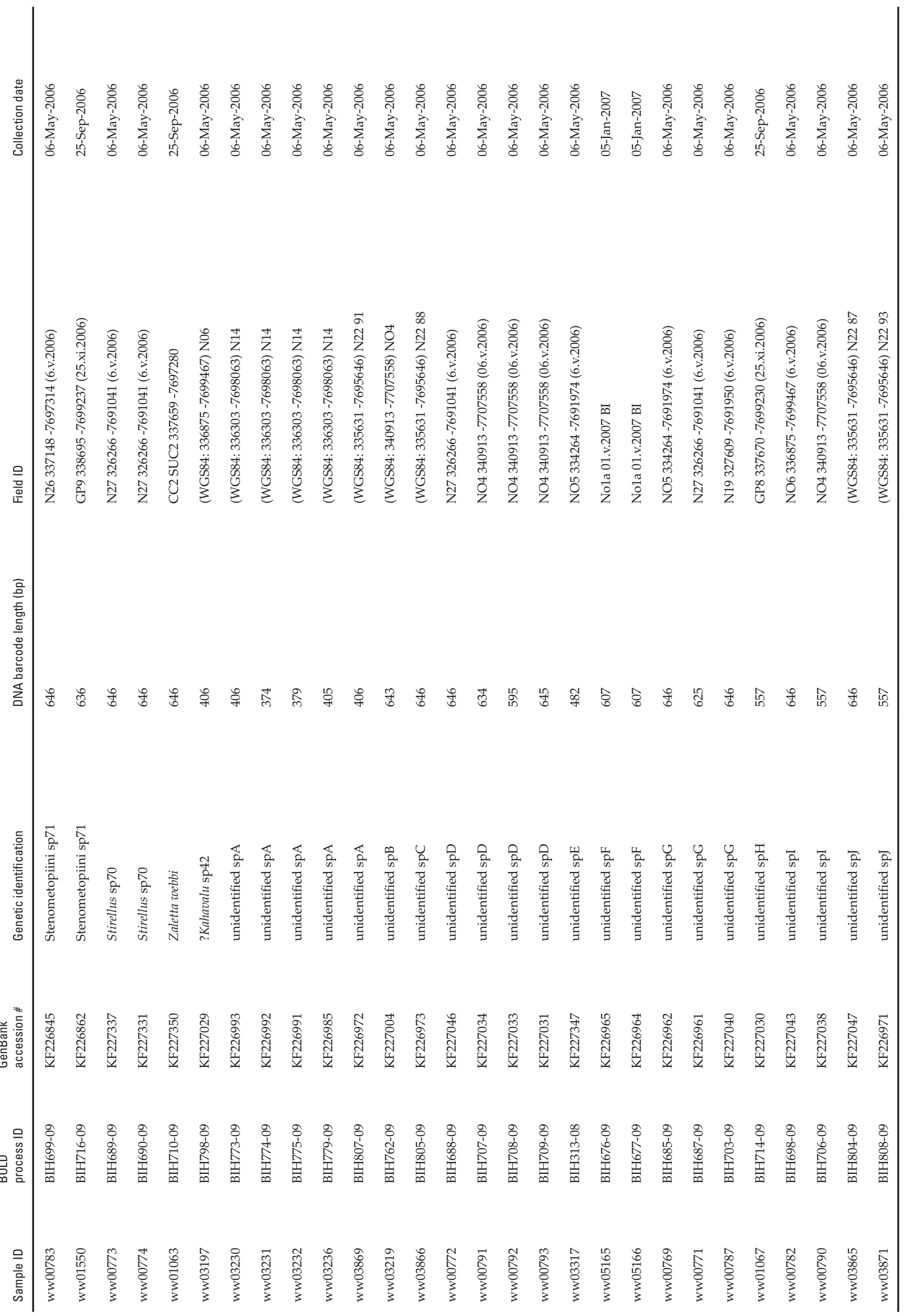

